JOURNAL OF

FUNCTION SPACES AND APPLICATIONS

Volume 2, Number 3 (2004), 323-356
(C) 2004, Scientific Horizon

http://www.jfsa.net

\title{
Local Uniform Convexity and Kadec-Klee Type Properties in $K$-interpolation spaces II
}

\author{
Peter G. Dodds*, Theresa K. Dodds*, Alexander A. Sedaev ${ }^{\dagger}$ and \\ Fyodor A. Sukochev*
}

(Communicated by Evgueni Semenov)

2000 Mathematics Subject Classification. Primary 46B70,46E30,46L52. Secondary 46B03,46B20.

Keywords and phrases. Lorentz couples, $K$-interpolation space, equivalent interpolation norms, local uniform convexity, symmetric Banach spaces of measurable operators.

\footnotetext{
Abstract. We study local uniform convexity and Kadec-Klee type properties in $K$-interpolation spaces of Lorentz couples. We show that a wide class of Banach couples of (commutative and) non-commutative Lorentz spaces possess the (so-called) ( $D G L$ )-property originally introduced by Davis, Ghoussoub and Lindenstrauss in the context of renorming order continuous Banach lattices. This property is used as a key tool to show that local uniform convexity and certain Kadec-Klee type properties in non-commutative symmetric spaces of measurable operators may be inferred from corresponding properties of the parameter space of the $K$-interpolation method. Further applications are given to renorming properties of separable symmetric Banach function spaces and their non-commutative counterparts.
}

\footnotetext{
*Research supported by the Australian Research Council.

${ }^{\dagger}$ Research supported by RFBR grant 02-01-00146 and by the Scientific program "Universities of Russia" Grant UR 04.01.051.
} 


\section{Introduction}

A Banach space $E$ is said to have the Kadec-Klee property (sometimes called the Radon-Riesz property) if weak and norm convergence of sequences coincide on the unit sphere. A closely related property is that of local uniform convexity, sometimes referred to as local uniform rotundity: $x, x_{n} \in$ $E, n=1,2, \ldots,\left\|x_{n}\right\|_{E} \rightarrow\|x\|_{E}$ and $\left\|x+x_{n}\right\|_{E} \rightarrow 2\|x\|_{E}$ imply that $x_{n} \rightarrow x$ in the norm of $E$. This paper is a continuation of the work in [16], which gave a detailed analysis of properties of a Banach couple $\mathbf{E}$ and properties of a $K$-interpolation functional $\Phi$ which guarantee that a $K$-interpolation space $\mathbf{E}_{\Phi}$ is locally uniformly convex or has some Kadec-Klee property. In this analysis, an important role is played by a key property of a general Banach couple $\mathbf{E}$, called the $(D G L)$-property. This property first appeared implicitly in [17] in the special case of the Banach couple $\left(L_{1}, L_{\infty}\right)(\Omega, P)$, with $(\Omega, P)$ a probability space and was used by them in a crucial way to prove that an order continuous Banach lattice admits an equivalent locally uniformly convex lattice norm. Essentially the same property arose implicitly in the work of Chilin, Krygin and Sukochev [8] in the more general setting of non-commutative couples of measurable operators to show that local uniform convexity could be "lifted" from an arbitrary symmetric Banach function space on the positive semi-axis to its non-commutative counterpart.

A principal result of this paper (Theorem 3.6) shows that, subject to natural restrictions, an arbitrary couple of (commutative and) noncommutative Lorentz spaces has the $(D G L)$-property. While containing the original assertion of [17] in the case of the Lorentz couple $\left(L_{1}[0,1), L_{\infty}[0,1)\right)$, this result is new for the special cases given by the commutative couples $\left(L_{1}[0, \infty), L_{\infty}[0, \infty)\right),\left(l_{1}(\mathbb{N}), l_{\infty}(\mathbb{N})\right)$ and as well for the non-commutative couple $\left(\mathcal{C}_{1}, \mathcal{C}_{\infty}\right)$, where $\mathcal{C}_{1}, \mathcal{C}_{\infty}$ respectively denote the Schatten ideals of trace-class operators and compact operators on some separable Hilbert space. Combined with the general theorems of [16], this now provides a unified framework to verify directly from properties of the underlying parameter space that certain concrete interpolation spaces for each of the basic $K$-interpolation functionals studied in [16] have Kadec-Klee properties or are locally uniformly convex (Corollary $3.7,3.10$ ). Our methods provide a new and unified approach to showing that local uniform convexity and various Kadec-Klee properties lift from a given symmetric space on the positive semi-axis to the corresponding non-commutative space. In particular, we extend results of [8], [6] to the setting of general noncommutative Lorentz couples.

In Section 4, we turn to the question of showing the existence of equivalent symmetric Kadec-Klee or locally uniformly convex norms on separable 
symmetric Banach function spaces on the positive semi-axis and their noncommutative counterparts. We give general criteria for a $K$-interpolation space to have an equivalent locally uniformly convex norm (Theorem 4.2) or to have an equivalent Kadec-Klee norm (Theorem 4.4). Using ideas that go back to [Se1,2], we show (Theorem 4.8, Corollary 4.10) that any separable symmetric Banach function space on the positive semi-axis admits an equivalent symmetric locally uniformly convex norm, as does its noncommutative counterpart. This is, of course, a natural refinement of the Kadec renorming theorem in the setting of symmetric Banach function spaces in that the equivalent norm is required to be symmetric. At the same time, it considerably extends the renorming theorem of [17] as the equivalent lattice norm given in [17] in the case of symmetric function spaces is a symmetric norm only in the case of symmetric spaces on the interval $[0,1)$.

Some of the results of this paper have been announced previously in [25].

\section{Preliminaries}

In the sequel, $\left(F,\|\cdot\|_{F}\right)$ will always denote a Banach function space on the measure space $(T, \mu)$, where $T$ is the interval $[0, \alpha)$ for some $0<\alpha \leq \infty$ and $\mu$ is Lebesgue measure $m$. Following [19] (see also [4]), a Banach function space (or ideal lattice) on $(T, \mu)$ is a Banach space $\left(F,\|\cdot\|_{F}\right)$ which is a linear subspace of the space $L^{0}(T, \mu)$ of all $\mu$-measurable, almost everywhere finite, complex-valued functions on $T$ which has the property that

$$
f \in F, g \in L^{0}(T, \mu),|g| \leq|f| \Longrightarrow g \in F \text { and }\|g\|_{F} \leq\|f\|_{F} .
$$

Unless stated otherwise, we shall always assume that the norm $\|\cdot\|_{F}$ on the Banach function space $F$ is a Fatou norm, that is, $\|\cdot\|_{F}$ is lower semicontinuous on $F$ for the topology $(l \mu)$ of local convergence in measure (that is, convergence in measure on every set of finite measure), in the sense that

$$
f, f_{n} \in F, \quad n=1,2, \ldots, \quad f_{n} \rightarrow_{l \mu} f \Longrightarrow\|f\|_{F} \leq \varliminf_{n}\left\|f_{n}\right\|_{F} .
$$

The norm on $F$ is said to be order continuous if and only if whenever $f_{n} \in F, n=1,2, \ldots$, satisfies $\left|f_{n}\right| \downarrow_{n} 0$, it follows that $\left\|f_{n}\right\|_{F} \rightarrow 0$.

If $f \in L^{0}(T, \mu)$, the non-increasing rearrangement $f^{*}$ of $f$ is defined by setting

$$
f^{*}(t)=\inf \{s: \mu\{|f|>s\}<t\}
$$


for all $t>0$. The rearrangement $f^{*}$ is non-increasing, right-continuous and is equimeasurable with $|f|$ in the sense that

$$
m\left\{f^{*}>t\right\}=\mu\{|f|>t\},
$$

for every $t \in \mathbb{R}^{+}$. The Banach function space $F$ on $(T, \mu)$ is said to be rearrangement-invariant (or symmetric, in the terminology of [19]) if and only if, whenever $g \in F$ and $f^{*} \leq g^{*}$, then $f \in F$ and $\|f\|_{F} \leq\|g\|_{F}$. We note that the familiar Orlicz, Lorentz and Marcinkiewicz spaces are rearrangement-invariant. For further details, we refer to [19], [4], [21]. We note, in particular, that the norm on a rearrangement-invariant Banach function space $F$ on $[0, \alpha), 0<\alpha \leq \infty$, with respect to Lebesgue measure $m$ is order continuous if and only if $F$ is separable.

If $f, g \in L^{0}(T, \mu)$ are locally integrable, we will write $f \prec g$ if and only if

$$
\int_{0}^{t} f^{*}(s) d s \leq \int_{0}^{t} g^{*}(s) d s
$$

for all $t>0$. If $F$ is a rearrangement-invariant Banach function space on $(T, \mu)$, then we will say that $F$ is fully symmetric if and only if $f \prec g, g \in F$ imply $f \in F$ and $\|f\|_{F} \leq\|g\|_{F}$.

Throughout this paper, we denote by $\mathbf{E}=\left(E_{0}, E_{1}\right)$ a Banach couple [5], [19], and set $\|\cdot\|_{i}=\|\cdot\|_{E_{i}}, i=0,1$. Let $S(\mathbf{E})$ and $I(\mathbf{E})$ denote the sum $E_{0}+E_{1}$ and the intersection $E_{0} \cap E_{1}$, respectively, with the usual norms:

$$
\begin{aligned}
\|x\|_{S(\mathbf{E})} & =\inf \left\{\left\|x^{0}\right\|_{0}+\left\|x^{1}\right\|_{1}: x=x^{0}+x^{1}, x^{0} \in E_{0}, x^{1} \in E_{1}\right\}, \\
\|x\|_{I(\mathbf{E})} & =\max \left\{\|x\|_{0},\|x\|_{1}\right\} .
\end{aligned}
$$

The $K$-functional $K(t, x ; \mathbf{E})$ of J. Peetre is defined for $x \in S(\mathbf{E})$ and $t>0$ by setting

$$
\begin{aligned}
K(t, x ; \mathbf{E}) & =K\left(t, x ; E_{0}, E_{1}\right) \\
& =\inf \left\{\left\|x^{0}\right\|_{0}+t\left\|x^{1}\right\|_{1}: x=x^{0}+x^{1}, x^{0} \in E_{0}, x^{1} \in E_{1}\right\} .
\end{aligned}
$$

It will be sometimes convenient to write $K(t, x)=K(t, x ; \mathbf{E})$ for brevity, and this should cause no confusion in the sequel. For each $x \in S(\mathbf{E})$, the function $K(\cdot, x)$ is concave and for every fixed $t>0$, the $K$-functional $K(t, \cdot)$ is a norm on $S(\mathbf{E})$, equivalent to the given norm of $S(\mathbf{E})$. This follows immediately by observing that

$$
\min (1, t)\|x\|_{S(\mathbf{E})} \leq K(t, x) \leq \max (1, t)\|x\|_{S(\mathbf{E})},
$$

for all $t>0$ and $x \in S(\mathbf{E})$. 
Let $E$ be a linear subspace of $S(\mathbf{E})$ and let $\emptyset \neq T \subseteq(0, \infty)$. The Banach couple $\mathbf{E}$ is said to have property $\left(C_{\mathcal{T}}\right)$ on the linear subspace $E$, written $\mathbf{E} \in\left(C_{\mathcal{T}} \mid E\right)$ relative to $T$, if and only if whenever $x_{n}, x \in E$ satisfy $x_{n} \rightarrow_{\mathcal{T}} x$ then it follows that

$$
K(t, x) \leq \underline{\lim }_{n} K\left(t, x_{n}\right) .
$$

$\mathbf{E}$ is said to have property $D_{\mathcal{T}}$ on the subspace $E$, written $\mathbf{E} \in\left(D_{\mathcal{T}} \mid E\right)$, relative to $T$ if and only if whenever $x_{n}, x \in E$ satisfy $x_{n} \rightarrow_{\mathcal{T}} x$ and $K\left(t, x_{n}\right) \rightarrow K(t, x)$ for all $t \in T$ then it follows that

$$
x_{n} \longrightarrow{ }_{S(\mathbf{E})} x .
$$

$\mathbf{E}$ is said to have property $(D G L)$ on the subspace $E$, written $\mathbf{E} \in(D G L \mid E)$, relative to $T$ if and only if whenever $x, x_{n} \in E, n=1,2, \ldots$, satisfy

$$
K\left(t, x_{n}\right) \longrightarrow_{n} K(t, x), \quad K\left(t, x+x_{n}\right) \longrightarrow_{n} 2 K(t, x)
$$

for all $t \in T$, it follows that $x_{n} \rightarrow x$ in $S(\mathbf{E})$. Finally, the Banach space $(E,\|\cdot\|)$ is said to have the Kadec-Klee property with respect to the topology $\mathcal{T}$ if $x_{n}, x \in E, n=1,2, \ldots, x_{n} \rightarrow_{\mathcal{T}} x$ and $\left\|x_{n}\right\| \rightarrow\|x\|$ imply that $x_{n} \rightarrow_{E} x$.

We shall need the following straightforward analogue for Banach couples of ([16] Proposition 1.3).

Proposition 2.1. Let $\mathbf{E}$ be a Banach couple and $E$ be a subspace of $S(\mathbf{E})$. If $\mathbf{E} \in(D G L \mid E)$ and $\mathbf{E} \in\left(C_{\mathcal{T}} \mid E\right)$ relative to the non-empty subset $T \subseteq(0, \infty)$ then $\mathbf{E} \in\left(D_{\mathcal{T}} \mid E\right)$ relative to $T$.

We recall (see [4], Chapter 5.1) that the space $E_{0}+\infty E_{1}$ (known as the Gagliardo completion of $E_{0}$ ) consists of all $x \in S(\mathbf{E})$ for which the norm

$$
K^{\infty}(x)=\sup _{0<t<\infty} K(t, x)=\lim _{t \rightarrow \infty} K(t, x)
$$

is finite. We remark that if the unit ball of $E_{0}$ is sequentially closed in $S(\mathbf{E})$, then $E_{0}+\infty E_{1}=E_{0}$. See ([4], Chapter 5, Theorem 1.4).

A Banach couple $\mathbf{E}$ will be said to have property $\left(D^{\infty}\right)$, written $\mathbf{E} \in\left(D^{\infty}\right)$ if and only if whenever $x_{n}, x \in S(\mathbf{E}), n=1,2, \ldots$, satisfy $x_{n} \rightarrow x \in S(\mathbf{E})$ and $K^{\infty}\left(x_{n}\right) \rightarrow K^{\infty}(x)$, it follows that

$$
K^{\infty}\left(x_{n}-x\right) \rightarrow 0
$$

We recall [16] the notion of a $K$-interpolation functional. Let $\mathcal{Q}$ be a cone of non-negative, increasing functions on $\mathbb{R}^{+}$containing the cone $Q$ of concave functions. A functional $\Phi: \mathcal{Q} \rightarrow[0,+\infty]$ is called a $K$-interpolation functional on the cone $\mathcal{Q}$ if 
(a) $\Phi$ is subadditive and positively homogeneous on $\mathcal{Q}$, that is,

$$
\Phi(f+g) \leq \Phi(f)+\Phi(g), \quad \Phi(\lambda f)=\lambda \Phi(f)
$$

for all $f, g \in \mathcal{Q}$ and $\lambda \geq 0$

(b) $\Phi$ is monotone on $\mathcal{Q}$, that is, if $g, f \in \mathcal{Q}$ and $g(t) \leq f(t)$ for all $t>0$, then

$$
\Phi(g) \leq \Phi(f)
$$

(c) $\Phi$ is non-trivial in the sense that, if $\Delta(t)=\min (1, t), t>0$, then $0<\Phi(\Delta)<\infty$.

Let $\Phi$ be a $K$-interpolation functional on the cone $\mathcal{Q}$. The functional $\Phi$ is said to be

(a) order-continuous on $\mathcal{Q}$ if and only if

$$
f, f_{n} \in \mathcal{Q}, f_{n}(t) \rightarrow 0 \text { for all } t>0, \Phi(f)<\infty \Longrightarrow \Phi\left(\min \left(f, f_{n}\right)\right) \rightarrow 0 ;
$$

(b) lower semicontinuous on $\mathcal{Q}$ if and only if

$$
f_{n}, f \in \mathcal{Q}, f_{n}(t) \rightarrow f(t) \text { for all } t>0, \Phi(f)<\infty \Longrightarrow \Phi(f) \leq \varliminf_{n} \Phi\left(f_{n}\right) .
$$

Let $\mathbf{E}=\left(E_{0}, E_{1}\right)$ be a Banach couple and $\Phi$ be a $K$-interpolation functional on $Q$. We set

$$
\mathbf{E}_{\Phi}=\{x \in S(\mathbf{E}): \Phi(K(\cdot, x))<\infty\} .
$$

Equipped with the norm

$$
\|x\|_{\Phi}=\Phi(K(\cdot, x)), \quad x \in \mathbf{E}_{\Phi},
$$

the space $\mathbf{E}_{\Phi}$ is an interpolation space with respect to the couple $\mathbf{E}$ with constant 1 . We shall refer to the space $\left(\mathbf{E}_{\Phi},\|\cdot\|_{\Phi}\right)$ as a $K$-interpolation space.

We recall two basic methods of constructing $K$-interpolation functionals. For further details, see [16]. Let $F$ be a Banach function space on $(T, \mu)$ and let $\rho(t), t \in T$ be a positive weight. Let $\mathcal{Q}$ be the cone generated by all functions of the form $h=|f-g|$, where $f, g$ are quasiconcave functions. We define the functional $\Phi_{\rho, F}$ on the cone $\mathcal{Q}$ by setting

$$
\Phi_{\rho, F}(f)=\left\|f \rho \chi_{T}\right\|_{F}, \quad f \in \mathcal{Q},
$$

where $\chi_{D}$ is the indicator function of the measurable set $D$. If $0<$ $\left\|\Delta \rho \chi_{T}\right\|_{F}<\infty$, then $\Phi_{\rho, F}$ is a $K$-interpolation functional on $\mathcal{Q}$. If $\mathbf{E}$ 
is a Banach couple, we set

$$
\mathbf{E}_{\rho, F}=\mathbf{E}_{\Phi_{\rho, F}}
$$

for notational simplicity and observe that $\mathbf{E}_{\rho, F}$ is a Banach space. This construction yields, as an important special case, the real interpolation spaces $\mathbf{E}_{\theta, p}, 0<\theta<1,1 \leq p<\infty$, (see [5]) consisting of all $x \in S(\mathbf{E})$ for which the functional

$$
\|x\|_{\theta, p}=\left\{\int_{0}^{\infty}\left[t^{-\theta} K(t, x ; \mathbf{E})\right]^{p} \frac{d t}{t}\right\}^{1 / p}
$$

is finite.

The theorem which follows is proved in ([16], Theorems 4.3, 4.7). We recall (see [7]) that the norm $\|\cdot\|_{F}$ on the Banach function space $F$ is said to be strictly monotone if and only if

$$
x, y \in F, \quad 0 \leq x \leq y, \quad x \neq y \Longrightarrow\|x\|_{F}<\|y\|_{F} .
$$

Theorem 2.2. Let $\left(F,\|\cdot\|_{F}\right)$ be a Banach function space on the measure space $(T, \mu)$ where $T$ is the interval $(0, \alpha)$ for some $0<\alpha \leq \infty$ and $\mu$ is Lebesgue measure $m$. Let $\rho$ be a strictly positive weight on $T$ and let $\mathbf{E}$ be a Banach couple.

(a) Let $\mathcal{T}$ be a Hausdorff linear topology on $S(\mathbf{E})$ weaker than the norm topology. If

(i) F has the Kadec-Klee property for local convergence in measure and has strictly monotone norm,

(ii) $\mathbf{E} \in\left(D_{\mathcal{T}} \mid \mathbf{E}_{\rho, F}\right)$ relative to $T$ and $\mathbf{E} \in\left(C_{\mathcal{T}} \mid \mathbf{E}_{\rho, F}\right)$ relative to $\mathbb{R}^{+}$, then $\mathbf{E}_{\rho, F}$ has the Kadec-Klee property with respect to $\mathcal{T}$.

(b) If $F$ is locally uniformly convex, and if $\mathbf{E} \in\left(D G L \mid \mathbf{E}_{\rho, F}\right)$ relative to $T$, then $\mathbf{E}_{\rho, F}$ is locally uniformly convex.

We now turn to the second principal method for constructing $K$ interpolation functionals. If $G$ is a fully symmetric space on $[0, \alpha), 0<$ $\alpha \leq \infty$, we define the $K$-interpolation functional $\Phi_{G}$ on the cone $Q$ of concave functions by setting

$$
\Phi_{G}(f)= \begin{cases}\left\|f^{\prime}\right\|_{G}, & \text { if } f \in Q_{0}, f^{\prime} \in G \\ \infty, & \text { otherwise, }\end{cases}
$$

for all $f \in Q$. See, for example, ([4] Definition 5.1.18). In the sequel, we shall denote the space $\mathbf{E}_{\Phi_{G}}$ by $\mathbf{E}_{G}$ and its norm by $\|\cdot\|_{\mathbf{E}_{G}}$. We remark that if $\mathcal{C}_{1}, \mathcal{C}_{\infty}$ denote the ideals of trace class and compact operators respectively 
on some separable Hilbert space, if $\mathbf{E}$ is the Banach couple $\left(\mathcal{C}_{1}, \mathcal{C}_{\infty}\right)$, and if $G=L_{p}[0, \infty), 1<p<\infty$, then the interpolation space $\mathbf{E}_{G}$ is the (noncommutative) Schatten ideal $\mathcal{C}_{p}$ consisting of all operators $x$ for which the sequence $\left\{\mu_{n}(x)\right\}_{n=1}^{\infty}$ of singular values of $x$ is an element of $l_{p}$ with norm given by

$$
\|x\|_{\mathcal{C}_{p}}=\left\|\left\{\mu_{n}(x)\right\}_{n=1}^{\infty}\right\|_{l_{p}} .
$$

More generally, if $(\mathcal{M}, \tau)$ is a semi-finite von Neumann algebra on some Hilbert space, if $\mathbf{E}$ is the Banach couple $\left(L_{1}(\mathcal{M}, \tau), \mathcal{M}\right)$ and if $G$ is a fully symmetric Banach function space on $\mathbb{R}^{+}$, then the interpolation space $\mathbf{E}_{G}$ is the non-commutative space $G(\mathcal{M}, \tau)$ corresponding to $G$.

The theorem which follows is proved in ([16], Theorems 4.6, 4.10). We recall [7] that the norm $\|\cdot\|_{G}$ on the symmetric Banach function space $G$ is said to be strictly $K$-monotone if and only if

$$
x, y \in G, \quad x \prec y, \quad x^{*} \neq y^{*} \Rightarrow\|x\|_{G}<\|y\|_{G} .
$$

Theorem 2.3. Let $G$ be a fully symmetric Banach function space on $(0, \alpha), 0<\alpha \leq \infty$.

(a) Let $\mathcal{T}$ be a Hausdorff linear topology on $S(\mathbf{E})$ weaker than the norm topology. Suppose that $G$ has the Kadec-Klee property for local convergence in measure and has strictly $K$-monotone norm, and that $\mathbf{E} \in\left(D_{\mathcal{T}} \mid \mathbf{E}_{G}\right)$ with respect to $T=(0, \alpha)$, and $\mathbf{E} \in\left(C_{\mathcal{T}} \mid \mathbf{E}_{G}\right)$ with respect to $(0, \infty)$.

(i) If $0<\alpha<\infty$, or, if $\alpha=\infty$ and $G \nsubseteq L_{1}[0, \infty)$, then $\mathbf{E}_{G}$ has the Kadec-Klee property with respect to $\mathcal{T}$.

(ii) If $\alpha=\infty$ and if either $E_{1} \subseteq E_{0}$ or $\mathbf{E} \in\left(D^{\infty}\right)$ then $\mathbf{E}_{G}$ has the Kadec-Klee property with respect to $\mathcal{T}$.

(b) Suppose that $G$ is locally uniformly convex and that $\mathbf{E} \in\left(D G L \mid \mathbf{E}_{G}\right)$ relative to $T=(0, \alpha)$.

(i) If $\alpha<\infty$, or, if $\alpha=\infty$ and $G \nsubseteq L_{1}$, then $\mathbf{E}_{G}$ is locally uniformly convex.

(ii) If $\alpha=\infty$ and if either $E_{1} \subseteq E_{0}$ or $\mathbf{E} \in\left(D^{\infty}\right)$ then $\mathbf{E}_{G}$ is locally uniformly convex.

In the sequel, we shall be almost exclusively concerned with the investigation of the properties $(D G L), D_{\mathcal{T}}, C_{\mathcal{T}}$ in Banach copules of commutative and non-commutative Lorentz spaces. We shall need the following facts and terminology drawn from the theory of non-commutative rearrangement invariant Banach function spaces.

Let $(\mathcal{M}, \tau)$ be a semifinite von Neumann algebra on the Hilbert space $\mathcal{H}$, with a fixed faithful and normal semifinite trace $\tau$ and identity $\mathbf{1}$. We denote by $\mathcal{M}^{p}$ the set of all projections in $\mathcal{M}$. A linear operator $x: \operatorname{dom}(x) \rightarrow \mathcal{H}$, 
with domain $\operatorname{dom}(x) \subseteq \mathcal{H}$, is called affiliated with $\mathcal{M}$ if $u x=x u$ for all unitary $u$ in the commutant $\mathcal{M}^{\prime}$ of $\mathcal{M}$. The closed and densely defined operator $x$, affiliated with $\mathcal{M}$, is called $\tau$-measurable if for every $\epsilon>0$ there exists $p \in \mathcal{M}^{p}$ such the $p(\mathcal{H}) \subseteq \operatorname{dom}(x)$ and $\tau(\mathbf{1}-p)<\epsilon$. With the sum and product defined as the respective closures of the algebraic sum and product, $\widetilde{\mathcal{M}}$ is a ${ }^{*}$-algebra. For $\epsilon, \delta>0$ we denote by $N(\epsilon, \delta)$ the set of all $x \in \widetilde{\mathcal{M}}$ for which there exists an orthogonal projection $p \in \mathcal{M}$ such that $p(\mathcal{H}) \subseteq \operatorname{dom}(x),\|x p\| \leq \epsilon$ and $\tau(1-p) \leq \delta$. The sets $\{N(\epsilon, \delta): \epsilon, \delta>0\}$ form a base at 0 for a metrizable Hausdorff topology in $\widetilde{\mathcal{M}}$, which is called the measure topology. Equipped with the measure topology, $\widetilde{\mathcal{M}}$ is a complete topological *-algebra. If a sequence in $\widetilde{\mathcal{M}}$ converges with respect to the measure topology we will also say that the sequence is convergent in measure. These facts and their proofs can be found in the papers [18], [22] and [29]. For standard facts concerning von Neumann algebras, we refer to [27], [28].

We recall the notion of generalized singular value function [18]. Given a self-adjoint operator $x$ in $\mathcal{H}$ we denote by $e^{x}(\cdot)$ the spectral measure of $x$. Now assume that $x \in \widetilde{\mathcal{M}}$. Then $e^{|x|}(B) \in \mathcal{M}$ for all Borel sets $B \subseteq \mathbb{R}$, and there exists $s>0$ such that $\tau\left(e^{|x|}(s, \infty)\right)<\infty$. For $x \in \widetilde{\mathcal{M}}$ and $t \geq 0$ we define

$$
\mu_{t}(x)=\inf \left\{s \geq 0: \tau\left(e^{|x|}(s, \infty)\right) \leq t\right\} .
$$

The function $\mu(x):[0, \infty) \rightarrow[0, \infty]$ is called the generalized singular value function (or non-increasing rearrangement) of $x$; note that $\mu_{t}(x)<\infty$ for all $t>0$. We mention in particular that a sequence $\left\{x_{n}\right\}_{n=1}^{\infty}$ in $\widetilde{\mathcal{M}}$ converges to 0 in measure if and only if $\mu_{t}\left(x_{n}\right) \rightarrow 0$ as $n \rightarrow \infty$, for all $t>0$.

Suppose that $\alpha>0$. If we consider $\mathcal{M}=L_{\infty}([0, \alpha), m)$, where $m$ denotes Lebesgue measure on the interval $[0, \alpha)$, as an Abelian von Neumann algebra acting via multiplication on the Hilbert space $\mathcal{H}=L^{2}([0, \alpha), m)$, with the trace given by integration with respect to $m$, it is easy to see that $\widetilde{\mathcal{M}}$ consists of all measurable functions on $[0, \alpha)$ which are bounded except on a set of finite measure. Further, if $f \in \widetilde{\mathcal{M}}$, then the generalized singular value function $\mu(f)$ is precisely the classical non-increasing rearrangement $|f|^{*}$ defined above. On the other hand, if $(\mathcal{M}, \tau)$ is the space of all bounded linear operators on some Hilbert space equipped with the canonical trace tr, then $\widetilde{\mathcal{M}}=\mathcal{M}$ and, if $x \in \mathcal{M}$ is compact, then the generalized singular value function $\mu(x)$ is given by

$$
\mu(x)=\sum_{n=0}^{\infty} \mu_{n}(x) \chi_{[n, n+1)},
$$


where $\left\{\mu_{n}(x)\right\}_{n=0}^{\infty}$ is the singular value sequence of $x$, that is, the eigenvalue sequence of $|x|=\sqrt{x^{*} x}$, arranged in non-increasing order and repeated according to multiplicity, and $\chi$ denotes the usual indicator function.

Given a semifinite von Neumann algebra $(\mathcal{M}, \tau)$ and a rearrangementinvariant Banach function space $\left(E,\|\cdot\|_{E}\right)$ on $([0, \tau(\mathbf{1})), m)$, we define the non-commutative space $E(\mathcal{M}, \tau)$ by setting

$$
E(\mathcal{M}, \tau):=\{x \in \widetilde{\mathcal{M}}: \mu(x) \in E\}, \quad\|x\|_{E(\mathcal{M}, \tau)}:=\|\mu(x)\|_{E} .
$$

We write $\mathcal{M}$ rather than $L_{\infty}(\mathcal{M}, \tau)$. If $E$ is symmetric, then the space $\left(E(\mathcal{M}, \tau),\|\cdot\|_{E(\mathcal{M}, \tau)}\right)$ is a Banach space and the inclusions

$$
L_{1}(\mathcal{M}, \tau) \cap \mathcal{M} \subseteq E(\mathcal{M}, \tau) \subseteq L_{1}(\mathcal{M}, \tau)+\mathcal{M}
$$

hold with continuous embeddings.

If $E$ is fully symmetric, then the space $E(\mathcal{M}, \tau)$ is an exact interpolation space for the Banach couple $\left(L_{1}(\mathcal{M}, \tau), \mathcal{M}\right)$. Further, if $E_{0}, E_{1}$ are fully symmetric Banach function spaces on $([0, \tau(\mathbf{1})), m)$, and if $\mathcal{F}$ is any exact interpolation functor, then

$$
\mathcal{F}\left(E_{0}, E_{1}\right)(\mathcal{M}, \tau)=\mathcal{F}\left(E_{0}(\mathcal{M}, \tau), E_{1}(\mathcal{M}, \tau)\right) .
$$

For details, we refer to [DDP2,3].

We write $E[0, \alpha)(=E)$ rather than $E\left(L_{\infty}[0, \alpha), m\right)$ if $\mathcal{M}$ is the commutative von Neumann algebra $L_{\infty}[0, \alpha)$ with trace given by integration with respect to Lebesgue measure, acting by multiplication on $L_{2}[0, \alpha)$, for some $0<\alpha \leq \infty$.

We set

$$
\widetilde{\mathcal{M}}_{0}:=\left\{x \in \widetilde{\mathcal{M}}: \mu_{t}(x) \rightarrow 0 \text { as } t \rightarrow \infty\right\}, \quad E_{0}(\mathcal{M}, \tau):=\widetilde{\mathcal{M}}_{0} \cap E(\mathcal{M}, \tau) .
$$

and let

$$
L_{0}(\mathcal{M}, \tau):=\widetilde{\mathcal{M}}_{0} \cap\left(L_{1}(\mathcal{M}, \tau)+\mathcal{M}\right) .
$$

We make the obvious remark that $\widetilde{\mathcal{M}}_{0}$ coincides with $\widetilde{\mathcal{M}}$ if $\tau(\mathbf{1})<\infty$. By ([14] Proposition 2.7), the closure of the space $L_{1}(\mathcal{M}, \tau) \cap \mathcal{M}$ in the space $L_{1}(\mathcal{M}, \tau)+\mathcal{M}$ coincides with $L_{0}(\mathcal{M}, \tau)$. It is clear that the embedding $E \subseteq L_{0}[0, \infty)$ implies the embedding $E(\mathcal{M}, \tau) \subseteq L_{0}(\mathcal{M}, \tau)$, and this embedding is continuous.

The Köthe dual $E(\mathcal{M}, \tau)^{\times}$is defined to be the set of all $x \in \widetilde{\mathcal{M}}$ such that $x y \in L_{1}(\mathcal{M}, \tau)$ for all $y \in E(\mathcal{M}, \tau)$. With the norm defined by setting

$\|x\|_{E(\mathcal{M}, \tau) \times}:=\sup \left\{\tau(|x y|): y \in E(\mathcal{M}, \tau),\|y\|_{E(\mathcal{M}, \tau)} \leq 1\right\}, \quad x \in E(\mathcal{M}, \tau)^{\times}$, 
the Köthe dual $E(\mathcal{M}, \tau)^{\times}$is a Banach space. Basic properties of Köthe duality, in the commutative setting may be found in [19], [4] (where the Köthe dual is called the associate space). In the non-commutative setting, the reader is referred to [14], where it is shown in particular that if $E$ is symmetric, then the space $\left(E(\mathcal{M}, \tau)^{\times},\|\cdot\|_{(E(\mathcal{M}, \tau) \times}\right)$ may be identified with the space $\left(E^{\times}(\mathcal{M}, \tau),\|\cdot\|_{E^{\times}(\mathcal{M}, \tau)}\right)$.

\section{Non-commutative Lorentz Couples}

Throughout this section, we shall be exclusively concerned with interpolation properties of Banach couples of (commutative and noncommutative) Lorentz spaces. We recall (see [19], Chapter II, section 5), that if $\phi$ is an increasing concave function on $[0, \infty)$ with $\phi(0)=0$, then the Lorentz space $\Lambda_{\phi}=\Lambda_{\phi}[0, \infty)$ consists of all Lebesgue measurable functions $x$ on $[0, \infty)$ for which

$$
\|x\|_{\Lambda_{\phi}}:=\int_{[0, \infty)} x^{*}(t) d \phi(t)<\infty .
$$

Here the integral is taken in the sense of Lebesgue-Stieltjes. For basic properties of Lorentz spaces, we refer to [19]. We note that the Lorentz space $\Lambda_{\phi}$ has order continuous norm if and only if $\phi(0+)=0$ and $\phi(\infty)=\infty$. We shall be concerned here with Banach couples of the form

$$
\Lambda^{\phi_{0}, \phi_{1}}=\left(\Lambda_{\phi_{0}}, \Lambda_{\phi_{1}}\right)
$$

where $\phi_{0}, \phi_{1}$ are concave functions whose quotient $\phi_{0} / \phi_{1}$ is increasing. Such couples play an important role in the interpolation theory of symmetric function spaces. See, for example, ([19] Chapter II.6), where the (so-called) main interpolation theorem is formulated in terms of Lorentz couples and their Marcinkiewicz counterparts. Let us observe that if $\phi_{0}(t)=t$ for all $t \geq 0$ and if $\phi_{1}(t)=1$ if $t>0$ and $\phi_{1}(0)=0$, then the Lorentz couple $\Lambda^{\phi_{0}, \phi_{1}}$ is the familiar Lebesgue couple

$$
\mathbf{L}[0, \infty)=\left(L_{1}[0, \infty), L_{\infty}[0, \infty)\right) .
$$

which in a certain sense provides a framework for the interpolation theory of symmetric spaces of measurable functions on the semi-axis.

A principal new result in this section shows that non-commutative Lorentz couples possess the $(D G L)$-property. This is given in Theorem 2.6 below. In the special case that the couple $\Lambda^{\phi_{0}, \phi_{1}}(\mathcal{M}, \tau)$ is the non-commutative couple

$$
\mathbf{L}(\mathcal{M}, \tau):=\left(L_{1}(\mathcal{M}, \tau), \mathcal{M}\right)
$$


this result was implicitly proved in ([8], Theorem 2.1). Our proof of Theorem 3.6 is based on their approach.

For brevity, we set

$$
\Lambda^{\phi_{0}, \phi_{1}}(\mathcal{M}, \tau):=\left(\Lambda_{\phi_{0}}(\mathcal{M}, \tau), \Lambda_{\phi_{1}}(\mathcal{M}, \tau)\right),
$$

and

$$
\Lambda_{0}^{\phi_{0}, \phi_{1}}(\mathcal{M}, \tau):=L_{0}(\mathcal{M}, \tau) \cap \Lambda_{\min \left(\phi_{0}, \phi_{1}\right)}(\mathcal{M}, \tau) .
$$

We shall need the following characterisation of convergence in measure which follows from ([8] Lemma 1.1).

Proposition 3.1. If $x_{n}, x \in \widetilde{\mathcal{M}}$, then the following statements are equivalent.

(i) $x_{n} \rightarrow x$ in measure.

(ii) $\left|x_{n}\right| \rightarrow|x|$ in measure and $\left|x_{n}+x\right| \rightarrow 2|x|$ in measure.

The interpolation formula which follows is an immediate consequence of combining ([19] Theorem II.5.9) and ([13], Theorem 3.2).

Lemma 3.2. If $\phi_{0}(s)$ and $\phi_{1}(s)$ are increasing concave functions whose quotient $\lambda(s):=\frac{\phi_{0}(s)}{\phi_{1}(s)}$ is increasing then, for every $x \in \Lambda_{\phi_{0}}(\mathcal{M}, \tau)+$ $\Lambda_{\phi_{1}}(\mathcal{M}, \tau)$,

$$
\begin{aligned}
K\left(t, x ; \Lambda^{\phi_{0}, \phi_{1}}(\mathcal{M})\right) & =K\left(t, \mu(x) ; \Lambda^{\phi_{0}, \phi_{1}}[0, \infty)\right) \\
& =\int_{0}^{\infty} \mu_{s}(x) d \min \left(\phi_{0}(s), t \phi_{1}(s)\right) \\
& =\int_{0}^{s(t)} \mu_{s}(x) d \phi_{0}(s)+t \int_{s(t)}^{\infty} \mu_{s}(x) d \phi_{1}(s),
\end{aligned}
$$

where for every $0<t<\infty$ the number $s(t)$ is uniquely determined from the condition $\lambda(s(t))=t$.

Lemma 3.3. If $x_{n}, x \in \Lambda_{0}^{\phi_{0}, \phi_{1}}(\mathcal{M}, \tau)$ for all $n \geq 1$ satisfy

$$
\left\|\mu\left(x_{n}\right)-\mu(x)\right\|_{\Lambda_{\min \left(\phi_{0}, t \phi_{1}\right)}[0, \infty)} \rightarrow 0
$$

then there exists a sequence $y_{n} \in \Lambda_{0}^{\phi_{0}, \phi_{1}}(\mathcal{M}, \tau)$ for all $n \geq 1$ such that $\mu\left(y_{n}\right)=\mu(x)$ for all $n \geq 1$ and

$$
\left\|y_{n}-x_{n}\right\|_{\Lambda_{0}^{\phi_{0}, \phi_{1}}(\mathcal{M}, \tau)} \rightarrow 0
$$

The proof of this Lemma is given in ([9], Lemma 7.3). 
The key idea which makes it possible to study the (DGL)-property for general Lorentz couples is given in the following Proposition, which is perhaps of interest in its own right.

Proposition 3.4. Let $\phi_{0}(s)$ and $\phi_{1}(s)$ be two increasing concave functions whose quotient $\phi_{0} / \phi_{1}$ is increasing, vanishes continuously at zero and satisfies $\lim _{s \rightarrow \infty} \phi_{0}(s) / \phi_{1}(s)=\infty$. If $x_{n}, x \in \Lambda_{0}^{\phi_{0}, \phi_{1}}(\mathcal{M}, \tau)$ for all $n \geq 1$ are such that

$$
K\left(t, x_{n} ; \Lambda^{\phi_{0}, \phi_{1}}(\mathcal{M}, \tau)\right) \rightarrow K\left(t, x ; \Lambda^{\phi_{0}, \phi_{1}}(\mathcal{M}, \tau)\right)
$$

and

$$
K\left(t, x_{n}+x ; \Lambda^{\phi_{0}, \phi_{1}}(\mathcal{M}, \tau)\right) \rightarrow 2 K\left(t, x ; \Lambda^{\phi_{0}, \phi_{1}}(\mathcal{M}, \tau)\right),
$$

for every $t>0$ as $n \rightarrow \infty$, then $\left\{x_{n}\right\}_{n=1}^{\infty}$ is convergent in measure to $x$.

Proof. By a standard argument [8], we may assume that the algebra $\mathcal{M}$ is non-atomic. By Proposition 2.1, it will suffice to show that $\left|x_{n}\right| \rightarrow|x|$ and $\left|x_{n}+x\right| \rightarrow 2|x|$ in measure. As the first step in this direction, we shall show that the sequences $\left\{\mu_{(\cdot)}\left(x_{n}\right)\right\}_{n=1}^{\infty}$ and $\left\{\frac{1}{2} \mu_{(\cdot)}\left(x_{n}+x\right)\right\}_{n=1}^{\infty}$ both converge to $\mu_{(\cdot)}(x)$ in measure. We note first that, for each $t>0$, the value of

$$
K\left(t, x ; \Lambda^{\phi_{0}, \phi_{1}}(\mathcal{M})\right)=K\left(t, \mu_{(\cdot)}(x) ; \Lambda^{\phi_{0}, \phi_{1}}(0, \infty)\right)
$$

coincides with the norm of element $x$ in the Lorentz space $\Lambda_{\min \left(\phi_{0}, t \phi_{1}\right)}(0, \infty)$ (see Lemma 3.2 above and ([19] Theorem II.5.9)). If $x \in \Lambda_{\min \left(\phi_{0}, t \phi_{1}\right)}[0, \infty)$, and $\alpha>0$, observe that

$$
\mu_{\alpha}(x)\left\|\chi_{[0, \alpha)}\right\|_{\Lambda_{\min \left(\phi_{0}, t \phi_{1}\right)}^{[0, \infty)}} \leq\|x\|_{\Lambda_{\min \left(\phi_{0}, t \phi_{1}\right)^{[0, \infty)}}} .
$$

From this, it immediately follows from the assumptions that for every $t>0$ the set $\left\{\mu_{t}\left(x_{n}\right)\right\}_{n=1}^{\infty}$ is bounded. Using Helly's principle and passing to a subsequence and relabelling if necessary, we may assume that the sequence $\left\{\mu_{(\cdot)}\left(x_{n}\right)\right\}_{n=1}^{\infty}$ converges to some non-increasing function $g$ almost everywhere on $[0, \infty)$. By Fatou's lemma, it follows that

$$
\int_{0}^{s(t)} g(s) d \phi_{0}(s)+t \int_{s(t)}^{\infty} g(s) d \phi_{1}(s)<\infty .
$$

Since for every $0<\alpha<\beta<\infty$ we have

$$
\int_{\alpha}^{\beta} \mu_{s}\left(x_{n}\right) d \phi_{i}(s) \rightarrow \int_{\alpha}^{\beta} g(s) d \phi_{i}(s), \quad i=0,1,
$$


and since for each $t>0$ ) we have, by assumption, that

$$
\begin{aligned}
\int_{0}^{s(t)} & \mu_{s}\left(x_{n}\right) d \phi_{0}(s)+t \int_{s(t)}^{\infty} \mu_{s}\left(x_{n}\right) d \phi_{1}(s) \\
& \rightarrow \int_{0}^{s(t)} \mu_{s}(x) d \phi_{0}(s)+t \int_{s(t)}^{\infty} \mu_{s}(x) d \phi_{1}(s),
\end{aligned}
$$

we see that

$$
\int_{0}^{s(t)} g(s) d \phi_{0}(s)+t \int_{s(t)}^{\infty} g(s) d \phi_{1}(s)=\int_{0}^{s(t)} \mu_{s}(x) d \phi_{0}(s)+t \int_{s(t)}^{\infty} \mu_{s}(x) d \phi_{1}(s) .
$$

Using the fact that

$$
t \phi_{1}(s(t))=\phi_{0}(s(t)), \quad t>0
$$

we obtain

$$
\phi_{1}(s(t))=\left[\phi_{0}^{\prime}(s(t))-t \phi_{1}^{\prime}(s(t))\right] s^{\prime}(t) .
$$

Consequently, taking derivatives of both sides of (3.1), we obtain that

$$
g(s(t)) \phi_{1}(s(t))+\int_{s(t)}^{\infty} g(s) d \phi_{1}(s)=\mu_{s(t)}(x) \phi_{1}(s(t))+\int_{s(t)}^{\infty} g(s) d \phi_{1}(s),
$$

or equivalently, with a simple change of notation, using the fact that the quotient $\phi_{0} / \phi_{1}$ has limit infinity at infinity,

$$
\left[g(s)-\mu_{s}(x)\right] \phi_{1}(s)=-\int_{s}^{\infty}\left[g(u)-\mu_{s}(x)\right] \phi_{1}^{\prime}(u) d u
$$

for all $s>0$. Again calculating derivatives, we obtain

$$
\frac{d}{d s}\left[\mu_{s}(x)-g(s)\right]=0
$$

for all $s>0$. Since $\lim _{s \rightarrow \infty} \mu_{s}(x)=0$ it follows that

$$
g(s)=\mu_{s}(x)+g(\infty)
$$

for all $s>0$, where $g(\infty)=\lim _{s \rightarrow \infty} g(s)$. If $g(\infty) \neq 0$, then substitution of (3.2) into (3.1) readily yields a contradiction. Consequently we obtain that the sequence $\left\{\mu\left(x_{n}\right)\right\}_{n=1}^{\infty}$ converges to $\mu(x)$ almost everywhere on $[0, \infty)$, and since $\mu\left(x_{n}\right), \mu(x), n=1,2, \ldots$, are non-increasing and vanish at infinity, it easily follows further that sequence $\left\{\mu\left(x_{n}\right)\right\}_{n=1}^{\infty}$ converges to $\mu(x)$ in measure. The assumption 


$$
K\left(t, x_{n}+x ; \Lambda^{\phi_{0}, \phi_{1}}(\mathcal{M}, \tau)\right) \rightarrow 2 K\left(t, x ; \Lambda^{\phi_{0}, \phi_{1}}(\mathcal{M}, \tau)\right),
$$

together with the same argument as in the previous paragraph shows that $\left\{\mu\left(\left(x_{n}+x\right) / 2\right)\right\}_{n=1}^{\infty}$ also converges to $\mu_{(\cdot)}(x)$ in measure. Since the Lorentz space $\Lambda_{\min \left(\phi_{0}, t \phi_{1}\right)}(0, \infty)$ has the Kadec-Klee property with respect to measure convergence (see [7], Corollary 1.3) we may now conclude that (3.3)

$\left\|\mu\left(x_{n}\right)-\mu(x)\right\|_{\Lambda_{\min \left(\phi_{0}, t \phi_{1}\right)}[0, \infty)} \rightarrow 0,\left\|\mu\left(\left(x_{n}+x\right) / 2\right) \mu(x)\right\|_{\Lambda_{\min \left(\phi_{0}, t \phi_{1}\right)}[0, \infty)} \rightarrow 0$.

From the first assertion of (3.3) combined with Lemma 3.3, there exists a sequence $y_{n} \in \Lambda_{0}^{\phi_{0}, \phi_{1}}(\mathcal{M}, \tau)$ for all $n \geq 1$ such that $\mu\left(y_{n}\right)=\mu(x)$ for all $n \geq 1$ for which

$$
\left\|y_{n}-x_{n}\right\|_{\Lambda_{0}^{\phi_{0}, \phi_{1}}(\mathcal{M}, \tau)} \rightarrow 0 .
$$

It follows immediately that

$$
\begin{gathered}
K\left(t, y_{n} ; \Lambda^{\phi_{0}, \phi_{1}}(\mathcal{M})\right) \rightarrow K\left(t, x ; \Lambda^{\phi_{0}, \phi_{1}}(\mathcal{M})\right), \\
K\left(t, y_{n}+x ; \Lambda^{\phi_{0}, \phi_{1}}(\mathcal{M})\right) \rightarrow 2 K\left(t, x ; \Lambda^{\phi_{0}, \phi_{1}}(\mathcal{M})\right),
\end{gathered}
$$

as $n \rightarrow \infty$. Therefore, without loss of generality, we may assume in addition that $\mu\left(x_{n}\right)=\mu(x)$, for all $n \geq 1$. Via the usual functional calculus, for each $\lambda>0$, we define the spectral projections $p(\lambda), q_{n}(\lambda), p_{n}(\lambda)$ by setting

$$
\begin{aligned}
p(\lambda):= & \chi_{(\lambda, \infty)}(|x|), \quad q_{n}(\lambda):=\chi_{(\lambda, \infty)}\left(\left|x_{n}\right|\right), \\
& p_{n}(\lambda):=\chi_{(\lambda, \infty)}\left(\left|\left(x_{n}+x\right) / 2\right|\right), \quad n \geq 1 .
\end{aligned}
$$

Since $x_{n}, x \in L_{0}(\mathcal{M}, \tau)$ it follows that $\tau(p(\lambda)), \tau\left(p_{n}(\lambda)\right), \tau\left(q_{n}(\lambda)\right)<\infty$ for all $n \geq 1$ and all $\lambda>0$. Since $\mu\left(x_{n}\right)=\mu(x)$, we have $\tau(p(\lambda))=\tau\left(q_{n}(\lambda)\right)$ and

$$
\tau(|x| p(\lambda))=\tau\left(\left|x_{n}\right| q_{n}(\lambda)\right), \quad n \geq 1, \quad \lambda>0 .
$$

Since $\left\{\frac{1}{2} \mu_{(\cdot)}\left(x_{n}+x\right)\right\}_{n=1}^{\infty}$ converges to $\mu_{(\cdot)}(x)$ in measure, we get by $([8]$, Lemma 1.5) that

$$
\tau\left(p_{n}(\lambda)\right) \rightarrow \tau(p(\lambda))
$$

for almost all $\lambda>0$. From (3.3) and the continuous embedding of $\Lambda_{\min \left(\phi_{0}, t \phi_{1}\right)}[0, \infty)$ into $\left(L_{1}+L_{\infty}\right)[0, \infty)$, it follows that

$$
\left\|\left(\frac{1}{2} \mu\left(x_{n}+x\right)-\mu(x)\right) \chi_{[0, \beta)}\right\|_{L_{1}(0, \beta)} \rightarrow 0
$$


for every $0<\beta<\infty$ and consequently

$$
\int_{0}^{\tau\left(p_{n}(\lambda)\right)} \frac{1}{2} \mu_{t}\left(x_{n}+x\right) d t \rightarrow \int_{0}^{\tau(p(\lambda))} \mu_{t}(x) d t
$$

for all $\lambda>0$ for which (3.4) holds. The argument of ([8] Theorem 2.1) now shows that

$$
\left\|p_{n}(\lambda)-p(\lambda)\right\|_{L_{1}(\mathcal{M}, \tau)} \rightarrow 0, \quad\left\|q_{n}(\lambda)-p(\lambda)\right\|_{L_{1}(\mathcal{M}, \tau)} \rightarrow 0 .
$$

By spectral approximation, it now follows that

$$
\left|x_{n}\right| \rightarrow|x|, \quad\left|x_{n}+x\right| \rightarrow 2|x|
$$

for the measure topology and the assertion that the sequence $\left\{x_{n}\right\}_{n=1}^{\infty}$ converges to $x$ for the measure topology now follows from Proposition 3.1.

Proposition 3.5. For any $\phi \in Q_{0}$, the non-commutative space $\Lambda_{\phi}(\mathcal{M}, \tau)$ has the Kadec-Klee property for convergence in measure.

In the setting of Lorentz spaces on the positive half-line, the preceding proposition is proved in ([7], Corollary 1.3). The principal technical ingredient is the submajorisation inequality given in ([19], Theorem II 3.1). Provided this inequality is replaced by its non-commutative extension given in ([12] Theorem 3.4), then the proof of [7] carries over from the commutative to the general non-commutative setting mutatis mutandi and we omit further details.

Theorem 3.6. If $\phi_{0}(s)$ and $\phi_{1}(s)$ are increasing concave functions whose quotient $\phi_{0} / \phi_{1}$ is increasing, vanishes continuously at zero and satisfies $\lim _{s \rightarrow \infty} \phi_{0}(s) / \phi_{1}(s)=\infty$, then $\Lambda^{\phi_{0}, \phi_{1}}(\mathcal{M}, \tau) \in\left(D G L \mid \Lambda_{0}^{\phi_{0}, \phi_{1}}(\mathcal{M}, \tau)\right)$ relative to $T=(0, \tau(\mathbf{1}))$.

Proof. Let $x_{n}, x \in \Lambda_{0}^{\phi_{0}, \phi_{1}}(\mathcal{M}, \tau)$ for all $n \geq 1$ satisfy the assumptions of Proposition 2.4. It will suffice to show that

$$
\left\|x-x_{n}\right\|_{\Lambda_{\min \left(\phi_{0}, \phi_{1}\right)}(\mathcal{M}, \tau)} \rightarrow 0 .
$$

The given assumptions together with Lemma 3.2 and the assertion of Proposition 3.4 imply that

$$
\left\|x_{n}\right\|_{\Lambda_{\min \left(\phi_{0}, \phi_{1}\right)}(\mathcal{M}, \tau)} \rightarrow\|x\|_{\Lambda_{\min \left(\phi_{0}, \phi_{1}\right)}(\mathcal{M}, \tau)}
$$

and that the sequence $\left\{x_{n}\right\}_{n=1}^{\infty}$ is convergent in measure to $x$. The desired conclusion now follows from the fact that the space $\Lambda_{\min \left(\phi_{0}, \phi_{1}\right)}(\mathcal{M}, \tau)$ 
has the Kadec-Klee property for convergence in measure, as follows from Proposition 3.5.

Remark. The result of Theorem 3.6 above is new even for classical Lorentz couples $\Lambda^{\phi_{0}, \phi_{1}}[0, \infty)$; in particular for the couple $\mathbf{L}[0, \infty)$ and for the couple $\mathbf{l}(\mathbb{N}):=\left(l_{1}, l_{\infty}\right)$. The $(D G L)$-property for the very special Lorentz couple

$$
\mathbf{L}[0,1):=\left(L_{1}[0,1), L_{\infty}[0,1)\right)
$$

was (again implicitly) introduced and studied in [17] and, as noted earlier, this is the source of the name for this property. It is shown in [17] that any separable symmetric Banach function space on $[0,1)$ admits an equivalent locally uniformly convex norm which, in addition, is symmetric. However, the lattice norms exhibited in [17] in the case of order continuous Banach lattices are not rearrangement invariant when specialised to the case of separable Banach function spaces on the positive semi-axis. We shall return to this question in the following section (see Corollary 4.9). Our approach here via Theorem 3.6 is completely different from the approach considered in [DGL1,2] and is applicable simultaneously in both commutative and noncommutative settings.

Corollary 3.7. Let $\phi_{0}, \phi_{1}$ be increasing concave functions whose quotient $\phi_{0} / \phi_{1}$ is increasing, vanishes continuously at zero and satisfies $\lim _{s \rightarrow \infty} \phi_{0}(s) / \phi_{1}(s)=\infty$. Let $\left(F,\|\cdot\|_{F}\right)$ be a Banach function space on the measure space $(T, \mu)$ where $T$ is the interval $(0, \tau(\mathbf{1}))$ and $\mu$ is Lebesgue measure $m$. Let $\rho$ be a strictly positive weight on $T$. If $F$ is locally uniformly convex and if $\Lambda^{\phi_{0}, \phi_{1}}(\mathcal{M}, \tau)_{\rho, F} \subseteq \Lambda_{0}^{\phi_{0}, \phi_{1}}(\mathcal{M}, \tau)$, then the Banach space $\Lambda^{\phi_{0}, \phi_{1}}(\mathcal{M}, \tau)_{\rho, F}$ is locally uniformly convex.

Proof. It follows immediately from Theorem 2.2(b), as the assumption that

$$
\Lambda^{\phi_{0}, \phi_{1}}(\mathcal{M}, \tau)_{\rho, F} \subseteq \Lambda_{0}^{\phi_{0}, \phi_{1}}(\mathcal{M}, \tau)
$$

together with Theorem 3.6 implies that

$$
\Lambda^{\phi_{0}, \phi_{1}}(\mathcal{M}, \tau) \in\left(D G L \mid \Lambda^{\phi_{0}, \phi_{1}}(\mathcal{M}, \tau)_{\rho, F}\right)
$$

with respect to $(0, \tau(\mathbf{1}))$.

Remark. Let us note that if

$$
\lim _{t \rightarrow \infty} \min \left\{\phi_{0}(t), \phi_{1}(t)\right\}=\infty
$$

then the Lorentz space

$$
\Lambda^{\phi_{0}, \phi_{1}}[0, \infty)=\Lambda_{\min \left\{\phi_{0}, \phi_{1}\right\}}[0, \infty)=\Lambda_{\phi_{0}}[0, \infty)+\Lambda_{\phi_{1}}[0, \infty)
$$


is separable. This implies [14] that

$$
\Lambda_{\phi_{0}}(\mathcal{M}, \tau)+\Lambda_{\phi_{1}}(\mathcal{M}, \tau)=\Lambda_{0}^{\phi_{0}, \phi_{1}}(\mathcal{M}, \tau)
$$

and consequently the condition $\Lambda^{\phi_{0}, \phi_{1}}(\mathcal{M}, \tau)_{\rho, F} \subseteq \Lambda_{0}^{\phi_{0}, \phi_{1}}(\mathcal{M}, \tau)$ of Corollary 3.7 is automatically satisfied in this case.

Proposition 3.8. The couple $\Lambda^{\phi_{0}, \phi_{1}}(\mathcal{M}, \tau)$ is a Gagliardo couple, that is

$$
\begin{aligned}
& \Lambda_{\phi_{0}}(\mathcal{M}, \tau)=\Lambda_{\phi_{0}}(\mathcal{M}, \tau)+\infty \Lambda_{\phi_{1}}(\mathcal{M}, \tau), \\
& \Lambda_{\phi_{1}}(\mathcal{M}, \tau)=\Lambda_{\phi_{1}}(\mathcal{M}, \tau)+\infty \Lambda_{\phi_{0}}(\mathcal{M}, \tau)
\end{aligned}
$$

Proof. Observe that if the sequence $\left\{x_{n}\right\} \subseteq \Lambda_{\phi_{0}}(\mathcal{M}, \tau)$ converges to $x$ in the norm of $\Lambda_{\phi_{0}}(\mathcal{M}, \tau)+\Lambda_{\phi_{1}}(\mathcal{M}, \tau)$ then sequence $\left\{x_{n}\right\}$ converges to $x$ in the measure topology, and so the sequence $\left\{\mu\left(x_{n}\right)\right\}$ converges to $\mu(x)$ almost everywhere on $[0, \infty)$. Consequently, if the norms of the sequence $\left\{x_{n}\right\}$ are bounded in the norm of the space $\Lambda_{\phi_{0}}(\mathcal{M}, \tau)$, then it follows from Fatou's Lemma that $\mu(x) \in \Lambda_{\phi_{0}}[0, \infty)$, and so $x \in \Lambda_{\phi_{0}}(\mathcal{M}, \tau)$. From this, it follows that

$$
\Lambda_{\phi_{0}}(\mathcal{M}, \tau)=\Lambda_{\phi_{0}}(\mathcal{M}, \tau)+\infty \Lambda_{\phi_{1}}(\mathcal{M}, \tau)
$$

and the equality

$$
\Lambda_{\phi_{1}}(\mathcal{M}, \tau)=\Lambda_{\phi_{1}}(\mathcal{M}, \tau)+\infty \Lambda_{\phi_{0}}(\mathcal{M}, \tau)
$$

is established by a similar argument.

Theorem 3.9. Suppose that $\phi_{0}(s)$ and $\phi_{1}(s)$ are increasing concave functions whose quotient $\phi_{0} / \phi_{1}(s)$ is increasing, vanishes continuously at zero and satisfies $\lim _{s \rightarrow \infty} \phi_{0}(s) / \phi_{1}(s)=\infty$. If $\tau(\mathbf{1})=\infty$, then $\Lambda^{\phi_{0}, \phi_{1}}(\mathcal{M}, \tau) \in\left(D^{\infty}\right)$.

Proof. Noting from Lemma 3.2 that

$$
\begin{aligned}
\|x\|_{\Lambda_{\phi_{0}}(\mathcal{M}, \tau)+\infty \Lambda_{\phi_{1}}(\mathcal{M}, \tau)} & =K^{\infty}(x)=\lim _{t \rightarrow \infty} K\left(t, x, \Lambda^{\phi_{0}, \phi_{1}}(\mathcal{M}, \tau)\right) \\
& =\|x\|_{\Lambda_{\phi_{0}}(\mathcal{M}, \tau)},
\end{aligned}
$$

we assume that $x, x_{n} \in \Lambda_{\phi_{0}}(\mathcal{M}, \tau)+\Lambda_{\phi_{1}}(\mathcal{M}, \tau)=\Lambda_{\min \left(\phi_{0}, \phi_{1}\right)}(\mathcal{M}, \tau), n=$ $1,2, \ldots$, that

$$
\left\|x_{n}-x\right\|_{\Lambda_{\min \left(\phi_{0}, \phi_{1}\right)}(\mathcal{M}, \tau)} \rightarrow 0
$$

and that

$$
\|x\|_{\Lambda_{\phi_{0}}(\mathcal{M}, \tau)}=K^{\infty}\left(x_{n}\right) \rightarrow K^{\infty}(x)=\|x\|_{\Lambda_{\phi_{0}}(\mathcal{M}, \tau)}<\infty .
$$


The first assertion implies that the sequence $\left\{x_{n}\right\}$ converges to $x$ for the measure topology. From the second assertion, it now follows from Proposition 3.5 that $x_{n} \rightarrow x$ in the space

$$
\Lambda_{\phi_{0}}(\mathcal{M}, \tau)=\Lambda_{\phi_{0}}(\mathcal{M}, \tau)+\infty \Lambda_{\phi_{1}}(\mathcal{M}, \tau)
$$

and so it now follows that $\Lambda^{\phi_{0}, \phi_{1}}(\mathcal{M}, \tau) \in\left(D^{\infty}\right)$.

Corollary 3.10. Suppose that $\phi_{0}(s)$ and $\phi_{1}(s)$ are increasing concave functions whose quotient $\phi_{0} / \phi_{1}$ is increasing, vanishes continuously at zero and satisfies $\lim _{s \rightarrow \infty} \phi_{0}(s) / \phi_{1}(s)=\infty$. If $G$ is a fully symmetric Banach function space on $[0, \tau(\mathbf{1}))$, if $G$ is locally uniformly convex, and if

$$
\Lambda^{\phi_{0}, \phi_{1}}(\mathcal{M}, \tau)_{G} \subseteq \Lambda_{0}^{\phi_{0}, \phi_{1}}(\mathcal{M}, \tau)
$$

then $\Lambda^{\phi_{0}, \phi_{1}}(\mathcal{M}, \tau)_{G}$ is locally uniformly convex.

Proof. If $0<\tau(\mathbf{1})<\infty$, then the Corollary is an immediate consequence of Theorem 2.3 (b) (i) and Theorem 3.6. If $\tau(\mathbf{1})=\infty$, then the Corollary follows from Theorem 2.3 (b)(ii), Theorem 3.6 and Theorem 3.9.

Corollary 3.11. Suppose that $\phi_{0}$ and $\phi_{1}$ are increasing concave functions whose quotient $\phi_{0} / \phi_{1}$ is increasing, vanishes continuously at zero and satisfies $\lim _{s \rightarrow \infty} \phi_{0}(s) / \phi_{1}(s)=\infty$. Let $G$ be a fully symmetric Banach function space on $[0, \tau(\mathbf{1}))$ and suppose that $G$ has the Kadec-Klee property for the weak topology on $G$ given by pointwise convergence on $L_{1}[0, \alpha) \cap$ $L_{\infty}[0, \alpha)$. If $\mathcal{T}$ is the weak topology on $\Lambda^{\phi_{0}, \phi_{1}}(\mathcal{M}, \tau)_{G}$ given by pointwise convergence on the space $L_{1}(\mathcal{M}, \tau) \cap L_{\infty}(\mathcal{M}, \tau)$ and if

$$
\Lambda^{\phi_{0}, \phi_{1}}(\mathcal{M}, \tau)_{G} \subseteq \Lambda_{0}^{\phi_{0}, \phi_{1}}(\mathcal{M}, \tau),
$$

then $\Lambda^{\phi_{0}, \phi_{1}}(\mathcal{M}, \tau)_{G}$ has the Kadec-Klee property with respect to $\mathcal{T}$.

Proof. The assumption that $G$ has the Kadec-Klee property for the topology on $G$ given by pointwise convergence on $L_{1}[0, \alpha) \cap L_{\infty}[0, \alpha)$ implies (and in fact is equivalent to) the statement that the norm on $G$ is strictly $K$-monotone and $G$ has the Kadec-Klee property for local convergence in measure. See ([7], Theorem 2.7). In particular, it follows from ([16], Proposition 2.6) that $G$ is separable. Note that, for each $t>0$, the $K$ functional $K(t, \cdot)$ is simply the norm on the space $\Lambda^{\phi_{0}}(\mathcal{M}, \tau)+$ $\Lambda^{t \phi_{1}}(\mathcal{M}, \tau)$. Since $L_{1}(\mathcal{M}, \tau) \cap L_{\infty}(\mathcal{M}, \tau) \subseteq \Lambda^{\phi_{0}}(\mathcal{M}, \tau) \cap \Lambda^{t \phi_{1}}(\mathcal{M}, \tau)$ is a norm-determining subset of the Köthe dual of $\Lambda^{\phi_{0}, \phi_{1}}(\mathcal{M}, \tau)_{G}$ by ([14], Proposition 2.5) (see also ([14], Theorem 2.6)), it follows readily that $\Lambda^{\phi_{0}, \phi_{1}}(\mathcal{M}, \tau) \in\left(C_{\mathcal{T}} \mid \Lambda^{\phi_{0}, \phi_{1}}(\mathcal{M}, \tau)_{G}\right)$ relative to any non-empty subset 
$T \subseteq(0, \infty)$. Using the assumption that

$$
\Lambda^{\phi_{0}, \phi_{1}}(\mathcal{M}, \tau)_{G} \subseteq \Lambda_{0}^{\phi_{0}, \phi_{1}}(\mathcal{M}, \tau)
$$

(which is automatically satisfied in the case that $\tau(\mathbf{1})<\infty$ ), it now follows from Theorem 2.6 and Proposition 1.1 that $\Lambda^{\phi_{0}, t \phi_{1}}(\mathcal{M}, \tau) \in$ $\left(D_{\mathcal{T}} \mid \Lambda^{\phi_{0}, t \phi_{1}}(\mathcal{M}, \tau)_{G}\right)$ relative to $(0, \tau(\mathbf{1})]$.

In the case that $\tau(\mathbf{1})<\infty$, it now follows from Theorem 2.3 (a)(i) that $\Lambda^{\phi_{0}, \phi_{1}}(\mathcal{M}, \tau)_{G}$ has the Kadec-Klee property with respect to $\mathcal{T}$. If $\tau(\mathbf{1})=\infty$, then Theorem 3.9 implies that $\Lambda^{\phi_{0}, \phi_{1}}(\mathcal{M}, \tau) \in\left(D^{\infty}\right)$. It now follows from Theorem 2.3(a)(ii) that $\Lambda^{\phi_{0}, t \phi_{1}}(\mathcal{M}, \tau)_{G}$ has the Kadec-Klee property with respect to $\mathcal{T}$ and this completes the proof of the Corollary.

Remark. In the special case that $\Lambda^{\phi_{0}, \phi_{1}}(\mathcal{M}, \tau)=\mathbf{L}(\mathcal{M}, \tau)$ the results of Corollary 3.10, Corollary 3.11, respectively, reduce to [8], Theorem 2.1 and [6], Theorem 2.8 respectively, which show that local uniform convexity and certain Kadec-Klee properties "lift" from a given symmetric Banach function space on the semi-axis to its non-commutative counterpart. Indeed, in this setting, the space $\mathbf{L}(\mathcal{M}, \tau)_{G}$ coincides with the non-commutative symmetric space $G(\mathcal{M}, \tau)$.

Corollary 3.12. Suppose that $\phi_{0}$ and $\phi_{1}$ are increasing functions whose quotient $\phi_{0} / \phi_{1}$ is increasing, vanishes continuously at zero and satisfies $\lim _{s \rightarrow \infty} \phi_{0}(s) / \phi_{1}(s)=\infty$. Let $\left(F,\|\cdot\|_{F}\right)$ be a separable symmetric Banach function space on the measure space $(T, \mu)$, where $T$ is the interval $(0, \tau(\mathbf{1}))$ and $\mu$ is Lebesgue measure $m$. Let $\rho$ be a strictly positive weight on T. If $F$ has the Kadec-Klee property for the topology given by pointwise convergence on $L_{1}(T, \mu) \cap L_{\infty}(T, \mu)$, if the norm on $F$ is strictly monotone and if

$$
\Lambda^{\phi_{0}, \phi_{1}}(\mathcal{M}, \tau)_{\rho, F} \subseteq \Lambda_{0}^{\phi_{0}, \phi_{1}}(\mathcal{M}, \tau)
$$

then $\Lambda^{\phi_{0}, \phi_{1}}(\mathcal{M}, \tau)_{\rho, F}$ has the Kadec-Klee property for the weak topology $\mathcal{T}$ given by pointwise convergence on the space $L_{1}(\mathcal{M}, \tau) \cap L_{\infty}(\mathcal{M}, \tau)$.

Proof. It follows from ([7] Theorem 2.7 (ii)) that $F$ has the Kadec-Klee property for local convergence in measure. By Theorem 2.2 (a), it will suffice to show that $\Lambda^{\phi_{0}, \phi_{1}}(\mathcal{M}, \tau) \in\left(D_{\mathcal{T}} \mid \Lambda^{\phi_{0}, \phi_{1}}(\mathcal{M}, \tau)_{\rho, F}\right)$ relative to $T$ and that $\Lambda^{\phi_{0}, \phi_{1}}(\mathcal{M}, \tau) \in\left(C_{\mathcal{T}} \mid \Lambda^{\phi_{0}, \phi_{1}}(\mathcal{M}, \tau)_{\rho, F}\right)$ relative to $T$. By Theorem 3.6 and Proposition 2.1, it will suffice to show only that $\Lambda^{\phi_{0}, \phi_{1}}(\mathcal{M}, \tau) \in$ $\left(C_{\mathcal{T}} \mid \Lambda^{\phi_{0}, \phi_{1}}(\mathcal{M}, \tau)_{\rho, F}\right)$ relative to $T$. To this end, suppose that $x_{n}, x \in$ $\Lambda^{\phi_{0}, \phi_{1}}(\mathcal{M}, \tau)_{\rho, F}, n=1,2, \ldots$, and that $x_{n} \rightarrow x$ pointwise on $L_{1}(\mathcal{M}, \tau) \cap$ $L_{\infty}(\mathcal{M}, \tau)$. Since $L_{1}(\mathcal{M}, \tau) \cap L_{\infty}(\mathcal{M}, \tau) \subseteq \Lambda^{\phi_{0}}(\mathcal{M}, \tau) \cap t \Lambda^{\phi_{1}}(\mathcal{M}, \tau)$ is normdetermining subset of the Köthe dual of

$$
\Lambda^{\phi_{0}, t \phi_{1}}(\mathcal{M}, \tau)=\Lambda_{\min \left(\phi_{0}, t \phi_{1}\right)}(\mathcal{M}, \tau),
$$


it follows via ([14], Proposition 2.5) that

$$
\begin{aligned}
K\left(t, x ; \Lambda^{\phi_{0}, \phi_{1}}(\mathcal{M}, \tau)\right) & =\|x\|_{\Lambda_{\min \left(\phi_{0}, t \phi_{1}\right)}(\mathcal{M}, \tau)} \leq \underline{\lim }_{n}\left\|x_{n}\right\|_{\Lambda_{\min \left(\phi_{0}, t \phi_{1}\right)}(\mathcal{M}, \tau)} \\
& =\underline{\lim }_{n} K\left(t, x_{n} ; \Lambda^{\phi_{0}, \phi_{1}}(\mathcal{M}, \tau)\right)
\end{aligned}
$$

for all $t>0$. Since $F$ is separable, it follows that the norm on $F$ is a Fatou norm, and, by ([16], Theorem 5.1), this readily implies that

$$
\Phi_{\rho, F}(x) \leq \liminf _{n \rightarrow \infty} \Phi_{\rho, F}\left(x_{n}\right) .
$$

This shows that $\Lambda^{\phi_{0}, \phi_{1}}(\mathcal{M}, \tau) \in\left(C_{\mathcal{T}} \mid \Lambda^{\phi_{0}, \phi_{1}}(\mathcal{M}, \tau)_{\rho, F}\right)$ relative to any nonempty subset of $(0, \infty)$ and suffices to complete the proof.

Remark. The result of the preceding Corollary continues to hold under the assumptions that $F$ is a Banach function space on the measure space $(T, \mu)$ with the Kadec-Klee property for local convergence in measure and with strictly monotone norm. The proof again follows directly from Theorem 2.2 by the same steps as above, with ([16], Proposition 1.2) ensuring that $F$ has Fatou norm.

\section{Renorming Theorems}

The $K$-interpolation functionals $\Phi, \Psi$ are said to be equivalent if there exist constants $0<c_{1}<c_{2}<\infty$ such that

$$
c_{1} \Phi(f) \leq \Psi(f) \leq c_{2} \Phi(f),
$$

for all $f \in Q$. In this section, we will study properties of an interpolation functional $\Phi$ and a Banach couple $\mathbf{E}$ which guarantee the existence of an equivalent interpolation functional $\Psi$ such that the interpolation norm $\|\cdot\|_{\Psi}$ is Kadec-Klee or locally uniformly convex.

Our discussion is centered on refinements of the Kadec theorem that each separable Banach space admits an equivalent locally uniformly convex norm. As noted in [17], such classical renormings in the case of Banach lattices or rearrangement-invariant Banach function spaces are almost never lattice norms, and certainly not rearrangement-invariant in the more restricted case of symmetric Banach function spaces on the semiaxis. One of the principal results of this section is a refinement of the Kadec renorming theorem which shows that any separable symmetric Banach function space on the positive semi-axis admits an equivalent locally uniformly convex norm which, in addition, is fully symmetric (Corollary 4.10$)$. We first note that the $(D G L)$ property of a given couple $\mathbf{E}$ may be considered as key to exhibiting the 
existence of equivalent locally uniformly convex norms. The Proposition which follows is proved in ([16], Proposition 2.11) and expresses the core idea in the approach of [17], in the case of the Lebesgue couple $\mathbf{L}[0,1)$.

Proposition 4.1. If $L \subseteq S(\mathbf{E})$ is a linear subspace, if $\emptyset \neq T \subseteq(0, \infty)$ and if $\mathbf{E} \in(D G L \mid L)$ relative to $T$, then the norm $\|\mid \cdot\|$ on $L$ defined by setting

$$
\|\| x \|=\left(\int_{0}^{\infty}\left[K(t, x) \min \left(1, t^{-2}\right]^{2} d t\right)^{1 / 2}, \quad x \in L,\right.
$$

is a locally uniformly convex norm on $L$ equivalent to that induced by $S(\mathbf{E})$.

It follows from Theorem 3.6 that the assumptions of the preceding Proposition are satisfied in the special case of any couple of the form $\mathbf{L}(\Omega, \mathcal{P})=\left(L_{1}(\Omega, \mathcal{P}), L_{\infty}(\Omega, \mathcal{P})\right)$ with $(\Omega, \mathcal{P})$ a probability space and $L=L_{1}(\Omega, \mathcal{P})$. Consequently, $L_{1}(\Omega, \mathcal{P})$ has an equivalent locally uniformly convex norm and this is precisely ([17], Theorem 1.1). Similarly, if $(\mathcal{M}, \tau)$ is a semifinite von Neumann algebra, then it follows from Theorem 3.6 that the assumptions of Proposition 4.1 are satisfied for the couple $\left(L_{1}(\mathcal{M}, \tau), \mathcal{M}\right)$ and the subspace $L=L_{0}(\mathcal{M}, \tau)$ (which is occasionally referred to in the literature as the algebra of $\tau$-compact operators). Consequently, the space $L=L_{0}(\mathcal{M}, \tau)$ admits an equivalent locally uniformly convex norm. As special cases, it follows that the familiar sequence space $c_{0}$ as well as the ideal $\mathcal{C}_{\infty}$ of all compact operators on some separable Hilbert space $\mathcal{H}$ equipped with the operator norm, admit equivalent locally uniformly convex norms. It is clear that these equivalent norms are fully symmetric. In the case of $c_{0}$, it is possibly not without interest to compare the norm given in the preceding proposition with Day's equivalent uniformly convex lattice norm on $c_{0}$, as presented in ([11], Chapter 4$)$.

We next give a general criterion for the existence of an equivalent locally uniformly convex $K$-interpolation norm. We need some additional terminology. We recall that a Banach space $X$ is said to be uniformly convex if and only if for all sequences $\left\{x_{n}\right\}_{n=1}^{\infty},\left\{y_{n}\right\}_{n=1}^{\infty} \subseteq X$,

$$
\left\|x_{n}\right\|_{X},\left\|y_{n}\right\|_{X} \rightarrow d,\left\|x_{n}+y_{n}\right\|_{X} \rightarrow 2 d \Longrightarrow\left\|x_{n}-y_{n}\right\|_{X} \rightarrow 0 .
$$

If $X$ is a Banach lattice, then $X$ is said to have the uniform strong majorant property if and only if for all sequences $\left\{x_{n}\right\}_{n=1}^{\infty},\left\{y_{n}\right\}_{n=1}^{\infty} \subseteq X$ such that $0 \leq x_{n} \leq y_{n}, n=1,2, \ldots$ and $\left\|x_{n}\right\|_{X},\left\|y_{n}\right\|_{X} \rightarrow d$, it follows that $\left\|x_{n}-y_{n}\right\|_{X} \rightarrow 0$. Let us note that if the Banach lattice $X$ is uniformly convex, then $X$ has the uniform strong majorant property. In fact, if $\left\{x_{n}\right\}_{n=1}^{\infty},\left\{y_{n}\right\}_{n=1}^{\infty} \subseteq X$ satisfy $0 \leq x_{n} \leq y_{n}, n=1,2, \ldots$ and 
$\left\|x_{n}\right\|_{X},\left\|y_{n}\right\|_{X} \rightarrow d$, then it follows from the inequalities

$$
2\left\|x_{n}\right\|_{X} \leq\left\|x_{n}+y_{n}\right\|_{X} \leq 2\left\|y_{n}\right\|_{X}
$$

that $\left\|x_{n}+y_{n}\right\|_{X} \rightarrow 2 d$. Uniform convexity of $X$ now implies that $\left\|x_{n}-y_{n}\right\|_{X} \rightarrow 0$ and consequently $X$ has the uniform strong majorant property.

Theorem 4.2. Let $\mathbf{E}$ be a Banach couple and let $\Phi$ be a $K$-interpolation functional on $Q$. If $\emptyset \neq T \subseteq(0, \infty)$ and if

(i) $\mathbf{E} \in\left(D G L \mid \mathbf{E}_{\Phi}\right)$ relative to $T$,

(ii) $\Phi$ is order-continuous and lower semicontinuous on $Q$

then there exists an interpolation functional $\Psi$ equivalent to $\Phi$ such that $\mathbf{E}_{\Psi}$ is locally uniformly convex.

Proof. For each $f \in Q$ and $t>0$, set

$$
f^{[n]}(t):=\min \left(f(t), \frac{t+1}{n}\right), \quad n=1,2, \ldots .
$$

We define the interpolation functional $\Psi$ by setting

$$
\Psi=\left(\Phi_{0}^{2}+\Phi_{1}^{2}+\Phi_{2}^{2}\right)^{1 / 2}
$$

where $\Phi_{0}=\Phi$,

$$
\Phi_{1}(f):=\sum_{n=1}^{\infty} 2^{-n} \Phi\left(f^{[n]}\right)
$$

and

$$
\Phi_{2}(f):=\left(\int_{0}^{\infty}\left(f(t) \min \left(1, t^{-2}\right)\right)^{2} d t\right)^{1 / 2},
$$

for all $f \in Q$. It is clear that $\Phi_{1}(f) \leq \Phi(f)$ for all $f \in Q$. Further, since

$$
\min (1, t) f(1) \leq f(t) \leq \max (1, t) f(1)
$$

for all $t>0$, it follows that

$$
\Phi_{2}(f) \leq f(1)\left(\int_{0}^{\infty}\left(\min \left(1, t^{-1}\right)\right)^{2} d t\right)^{1 / 2}, \quad f(1) \Phi(\min (\mathbf{1}, \cdot)) \leq \Phi(f) .
$$

This yields readily that

$$
\Phi_{2}(f) \leq c \Phi(f)
$$


for all $f \in Q$, with

$$
c=\frac{\left(\int_{0}^{\infty}\left(\min \left(1, t^{-1}\right)\right)^{2} d t\right)^{1 / 2}}{\Phi(\min (\mathbf{1}, \cdot))} .
$$

It follows immediately from these remarks that the functionals $\Phi, \Psi$ are equivalent. To show that $\mathbf{E}_{\Psi}$ is locally uniformly convex, suppose that $x_{n}, x, n=1,2, \cdots \in \mathbf{E}_{\text {क }}$ satisfy

$$
\Psi\left(K\left(\cdot, x_{n}\right)\right)=\left\|x_{n}\right\|_{\Psi} \rightarrow\|x\|_{\Psi}=\Psi(K(\cdot, x))
$$

and

$$
\Psi\left(K\left(\cdot, x_{n}+x\right)=\left\|x_{n}+x\right\|_{\Psi} \rightarrow 2\|x\|_{\Psi}=2 \Psi(K(\cdot, x) .\right.
$$

We define $\mathbf{a}, \mathbf{a}_{\mathbf{n}}, \mathbf{b}_{\mathbf{n}}, \mathbf{c}_{\mathbf{n}} \in l_{2}^{(3)}, n=1,2, \ldots$ by setting

$$
\begin{gathered}
\mathbf{a}=\left(\Phi_{0}(K(\cdot, x)), \Phi_{1}(K(\cdot, x)), \Phi_{2}(K(\cdot, x))\right), \\
\mathbf{a}_{\mathbf{n}}=\left(\Phi_{0}\left(K\left(\cdot, x_{n}\right)\right), \Phi_{1}\left(K\left(\cdot, x_{n}\right)\right), \Phi_{2}\left(K\left(\cdot, x_{n}\right)\right)\right),
\end{gathered}
$$

and

$$
\begin{aligned}
& \mathbf{b}_{\mathbf{n}}=\left(\Phi_{0}\left(K\left(\cdot, x+x_{n}\right)\right), \Phi_{1}\left(K\left(\cdot, x+x_{n}\right)\right), \Phi_{2}\left(K\left(\cdot, x+x_{n}\right)\right)\right) \\
& \mathbf{c}_{\mathbf{n}}=\left(\Phi_{0}\left(K(\cdot, x)+K\left(\cdot, x_{n}\right)\right), \Phi_{1}\left(K(\cdot, x)+K\left(\cdot, x_{n}\right)\right), \Phi_{2}\left(K(\cdot, x)+K\left(\cdot, x_{n}\right)\right)\right),
\end{aligned}
$$

and note that

$$
\|\mathbf{a}\|_{l_{2}^{(3)}}=\|x\|_{\Psi}, \quad\left\|\mathbf{a}_{\mathbf{n}}\right\|_{l_{2}^{(3)}}=\left\|x_{n}\right\|_{\Psi}, \quad\left\|\mathbf{b}_{\mathbf{n}}\right\|_{l_{2}^{(3)}}=\left\|x+x_{n}\right\|_{\Psi},
$$

so that

$$
\left\|\mathbf{a}_{\mathbf{n}}\right\|_{l_{2}^{(3)}} \rightarrow\|\mathbf{a}\|_{l_{2}^{(3)}}, \quad\left\|\mathbf{b}_{\mathbf{n}}\right\|_{l_{2}^{(3)}} \rightarrow 2\|\mathbf{a}\|_{l_{2}^{(3)}}
$$

Now observe that the inequalities

$$
\mathbf{0} \leq \mathbf{b}_{\mathbf{n}} \leq \mathbf{c}_{\mathbf{n}} \leq \mathbf{a}+\mathbf{a}_{\mathbf{n}}, \quad n=1,2, \ldots,
$$

hold with respect to the lattice ordering on $l_{2}^{(3)}$, and consequently

$$
\left\|\mathbf{b}_{\mathbf{n}}\right\|_{l_{2}^{(3)}} \leq\left\|\mathbf{c}_{\mathbf{n}}\right\|_{l_{2}^{(3)}} \leq\left\|\mathbf{a}+\mathbf{a}_{\mathbf{n}}\right\|_{l_{2}^{(3)}} \leq\|\mathbf{a}\|_{l_{2}^{(3)}}+\left\|\mathbf{a}_{\mathbf{n}}\right\|_{l_{2}^{(3)}}
$$

for $n=1,2, \ldots$ From this it follows that

$$
\left\|\mathbf{c}_{\mathbf{n}}\right\|_{l_{2}^{(3)}} \rightarrow 2\|\mathbf{a}\|_{l_{2}^{(3)}}, \quad\left\|\mathbf{a}+\mathbf{a}_{\mathbf{n}}\right\|_{l_{2}^{(3)}} \rightarrow 2\|\mathbf{a}\|_{l_{2}^{(3)}} .
$$


Using the fact that the norm on $l_{2}^{(3)}$ is uniformly convex and so has the uniform strong majorant property, it follows that

$$
\left\|\mathbf{a}_{\mathbf{n}}-\mathbf{a}\right\|_{l_{2}^{(3)}} \rightarrow 0, \quad\left\|\mathbf{a}_{\mathbf{n}}+\mathbf{a}-\mathbf{c}_{\mathbf{n}}\right\|_{l_{2}^{(3)}} \rightarrow 0 .
$$

The first limit implies that

$$
\Phi_{i}\left(K\left(\cdot, x_{n}\right)\right) \rightarrow \Phi_{i}(K(\cdot, x)),
$$

for $i=0,1,2$, and this assertion together with the second limit implies that

$$
\Phi_{i}\left(K\left(\cdot, x_{n}\right)+K(\cdot, x)\right) \rightarrow 2 \Phi_{i}(K(\cdot, x))
$$

for $i=0,1,2$. Using now the uniform convexity of the norm $\Phi_{2}$, this in turn implies that

$$
\Phi_{2}\left(K\left(\cdot, x_{n}\right)-K(\cdot, x)\right) \rightarrow 0
$$

and the same argument as in the proof of Proposition 4.1, based on the uniform strong majorant property of $\Phi_{2}$ shows further that

$$
\Phi_{2}\left(K\left(\cdot, x_{n}+x\right)-2 K(\cdot, x)\right) \rightarrow 0 .
$$

Passing to subsequences if necessary and relabelling, it now follows from the definition of $\Phi_{2}$ that

$$
K\left(\cdot, x_{n}\right) \rightarrow K(\cdot, x), \quad \text { and } \quad K\left(\cdot, x_{n}+x\right) \rightarrow 2 K(\cdot, x)
$$

locally in measure. Passing to a further subsequence if necessary, we may assume via ([16], Proposition 1.10) that

$$
K\left(t, x_{n}\right) \rightarrow K(t, x), \quad \text { and } \quad K\left(t, x_{n}+x\right) \rightarrow 2 K(t, x)
$$

for all $t>0$. By the assumption that $\mathbf{E} \in\left(D G L \mid \mathbf{E}_{\Phi}\right)$ relative to $\emptyset \neq T \subseteq(0, \infty)$, it now follows that $x_{n} \rightarrow x$ in the norm of $S(\mathbf{E})$. We now set $y_{n}:=x_{n}-x, n=1,2, \ldots$, and suppose that there exists $\epsilon>0$ such that

$$
\left\|y_{n}\right\|_{E_{\Phi}}>3 \epsilon, \quad n=1,2, \ldots .
$$

From the preceding argument, it follows that

$$
K\left(t, y_{n}\right)=K\left(t, x_{n}-x\right) \rightarrow 0 \quad \text { and } \quad K\left(t, x_{n}\right) \rightarrow K(t, x)
$$

for all $t>0$, and that

$$
K^{[m]}\left(\cdot, y_{n}\right)=K\left(\cdot, y_{n}\right)
$$

for each $m=1,2, \ldots$, and all sufficiently large $n$ (depending on $m$ ). Using the assumption that $\Phi$ is order continuous on $Q$, there exists $N \in \mathbb{N}$ such 
that

$$
\Phi\left(K^{[n]}(\cdot, x)\right)<\epsilon
$$

for all $n \geq N$. By the preceding remark, it may be assumed that

$$
\Phi\left(K^{[N]}\left(\cdot, y_{n}\right)\right)=\Phi\left(K\left(\cdot, y_{n}\right)\right)
$$

for all $n \geq N$. Noting that

$$
K^{[N]}\left(\cdot, y_{n}\right) \leq K^{[N]}\left(\cdot, x_{n}\right)+K^{[N]}(\cdot, x), \quad n=1,2, \ldots
$$

and using the subadditivity of $\Phi$, we obtain that

$$
\begin{aligned}
\Phi\left(K^{[N]}\left(\cdot, x_{n}\right)\right) & \geq \Phi\left(K^{[N]}\left(\cdot, y_{n}\right)\right)-\Phi\left(K^{[N]}(\cdot, x)\right) \\
& \geq \Phi\left(K\left(\cdot, y_{n}\right)\right)-\Phi\left(K^{[N]}(\cdot, x)\right) \\
& \geq 3 \epsilon-\Phi\left(K^{[N]}(\cdot, x)\right) \\
& >\epsilon+\Phi\left(K^{[N]}(\cdot, x)\right),
\end{aligned}
$$

for all $n \geq N$. Consequently

$$
\underline{\lim }_{n} \Phi\left(K^{[N]}\left(\cdot, x_{n}\right)\right)>\epsilon+\Phi\left(K^{[N]}(\cdot, x)\right) .
$$

Since $\Phi$ is lower semicontinuous on the cone $Q$ and since $K\left(t, x_{n}\right) \rightarrow K(t, x)$ for every $t>0$, it follows that

$$
\varliminf_{n} \Phi\left(K^{[i]}\left(\cdot, x_{n}\right)\right) \geq \Phi\left(K^{[i]}(\cdot, x)\right)
$$

for all $i \in \mathbb{N}$. Suppose now that $0<a, b, c \in \mathbb{R}$ satisfy $a^{2}+b^{2}+c^{2}=1$ and observe that

$$
\begin{aligned}
\lim _{n \rightarrow \infty} \Psi\left(K\left(\cdot, x_{n}\right)\right) & \geq \underline{\lim }_{n}\left[a \Phi\left(K\left(\cdot, x_{n}\right)\right)+b \Phi_{1}\left(K\left(\cdot, x_{n}\right)\right)+c \Phi_{2}\left(K\left(\cdot, x_{n}\right)\right)\right] \\
& \geq a \underline{\lim }_{n} \Phi\left(K\left(\cdot, x_{n}\right)\right)+b \sum_{i \neq N} 2^{-i} \underline{\lim }_{n} \Phi\left(K^{[i]}\left(\cdot, x_{n}\right)\right) \\
& +b 2^{-N} \underline{\lim }_{n} \Phi\left(K^{[N]}\left(\cdot, x_{n}\right)\right)+c \underline{\lim }_{n} \Phi_{2}\left(K\left(\cdot, x_{n}\right)\right) \\
& \geq a \Phi(K(\cdot, x))+b \Phi_{1}(K(\cdot, x))+c \Phi_{2}(K(\cdot, x))+b 2^{-N} \epsilon .
\end{aligned}
$$

If we now set

$$
a=\frac{\Phi(K(\cdot, x))}{\Psi(K(\cdot, x))}, \quad b=\frac{\Phi_{1}(K(\cdot, x))}{\Psi(K(\cdot, x))}, \quad c=\frac{\Phi_{2}(K(\cdot, x))}{\Psi(K(\cdot, x))},
$$


we obtain that

$$
\lim _{n \rightarrow \infty} \Psi\left(K\left(\cdot, x_{n}\right)\right) \geq \Psi(K(\cdot, x))+\epsilon 2^{-N} \frac{\Phi_{1}(K(\cdot, x))}{\Psi(K(\cdot, x))} .
$$

This contradicts the assumption that $\left\|x_{n}\right\|_{\Psi} \rightarrow\|x\|_{\Psi}$, and suffices to complete the proof of the Theorem.

Corollary 4.3. If $(\mathcal{M}, \tau)$ is a finite von Neumann algebra and if $E$ is a separable symmetric Banach function space on $[0, \tau(\mathbf{1}))$, then the space $E(\mathcal{M}, \tau)$ has an equivalent fully symmetric locally uniformly convex norm.

Proof. Let $\mathbf{L}=\mathbf{L}(\mathcal{M}, \tau)$ be the non-commutative couple $\left(L_{1}(\mathcal{M}, \tau), \mathcal{M}\right)$ and note that the space $E(\mathcal{M}, \tau)$ is just the interpolation space $\mathbf{L}_{E}$. It follows from Theorem 3.6 that $\mathbf{L}(\mathcal{M}, \tau) \in(D G L \mid E(\mathcal{M}, \tau))$ relative to $T=(0, \tau(\mathbf{1}))$. Since $E$ is separable, it follows that $E$ has order continuous norm, and this implies that $\Phi_{E}$ is order continuous on the cone $Q$ by ([16], Theorem 4.2 (i)). The Corollary now follows from Theorem 4.2.

The preceding Corollary is an exact non-commutative extension of ([17], Corollary 1.2). See also ([8], Corollary 2.2). In particular, it follows that any (commutative) separable symmetric Banach function space on a finite interval has an equivalent fully symmetric locally convex norm.

The theorem which follows is the counterpart to Theorem 4.2 for the Kadec-Klee property. The proof follows the same lines as that of Theorem 4.2 , with the role of the $(D G L)$-property replaced by the $\left(D_{\mathcal{T}}\right)$-property. The details are left to the interested reader.

Theorem 4.4. Let $\mathbf{E}=\left(E_{0}, E_{1}\right)$ be a Banach couple, let $\mathcal{T}$ be a Hausdorff linear topology on $S(\mathbf{E})$ weaker than the norm topology and let $\Phi$ be a $K$ interpolation functional on $Q$. If $\emptyset \neq T \subseteq(0, \infty)$ and if

(i) $\mathbf{E} \in\left(D_{\mathcal{T}} \mid \mathbf{E}_{\Phi}\right)$ relative to $T$ and $\mathbf{E} \in\left(C_{\mathcal{T}} \mid \mathbf{E}_{\Phi}\right)$ relative to $(0, \infty)$,

(ii) $\Phi$ is order-continuous and lower semicontinuous on $Q$.

then there exists an interpolation functional $\Psi$ equivalent to $\Phi$ such that $\mathbf{E}_{\Psi}$ has the Kadec-Klee property with respect to $\mathcal{T}$.

We will now show how the general Theorem 4.2 may be applied to obtain concrete renorming theorems in the case of explicit couples. Theorem 4.2 itself does not provide an immediate approach in the case that the interpolation functional is of the form $\Phi_{G}$ where $G$ is a separable fully symmetric space on $[0, \infty)$. In this case, it has been noted in [16] that such functionals, in general, are not order-continuous. Nonetheless, it is possible to vary the approach of Proposition 4.1 as follows.

Proposition 4.5. Let $\mathbf{E}$ be a Banach couple. Suppose that $\mathbf{E} \in D^{\infty}$ and that $\mathbf{E} \in\left(D G L \mid E_{0}\right)$ relative to $T$ where $\emptyset \neq T \subseteq(0, \infty)$. If $E_{0}$ is Gagliardo 
complete with respect to $\mathbf{E}$, that is, if $E_{0}=E_{0}+\infty E_{1}$ (with equivalent norms), then $E_{0}$ admits an equivalent locally uniformly convex norm.

Proof. For each $x \in E_{0}$, set

$$
\|\mid\| x \|:=\left(\left(K^{\infty}(x)\right)^{2}+\|K(\cdot, x)\|_{2}^{2}\right)^{1 / 2}
$$

where $K^{\infty}(x)=K(\infty, x)=\lim _{t \rightarrow \infty} K(t, x)$ and

$$
\|K(\cdot, x)\|_{2}=\left(\int_{0}^{\infty}\left[K(t, x) \min \left(1, t^{-2}\right)\right]^{2} d t\right)^{1 / 2} .
$$

From the assumption that $E_{0}$ is Gagliardo complete, it follows that the norm $\||\cdot|\|$ is equivalent to the given norm on $E_{0}$. Suppose that $x_{n}, x \in$ $E_{0}, n=1,2, \ldots$, satisfy

$$
\left\|x_{n}\right\|\|\rightarrow\| x\|\|, \quad\left\|\left|x+x_{n}\||\rightarrow 2|\| x\|\| .\right.\right.
$$

Repeating the arguments from the proof of Theorem 4.2 and using the uniform convexity and the uniform strong majorant property of the twodimensional $l_{2}$ norm, we obtain that

$$
K^{\infty}\left(x_{n}\right) \rightarrow K^{\infty}(x)
$$

that

$$
\left\|K\left(\cdot, x_{n}\right)\right\|_{2} \rightarrow\|K(\cdot, x)\|_{2}, \quad\left\|K\left(\cdot, x+x_{n}\right)\right\|_{2} \rightarrow 2\|K(\cdot, x)\|_{2},
$$

and that

$$
\left\|K\left(\cdot, x_{n}\right)+K(\cdot, x)\right\|_{2} \rightarrow 2\|K(\cdot, x)\|_{2} .
$$

Using the uniform convexity and the uniform strong majorant property of the $\|\cdot\|_{2}$, we obtain that

$$
K\left(t, x_{n}\right) \rightarrow K(t, x), \quad K\left(t, x+x_{n}\right) \rightarrow 2 K(t, x)
$$

for all $t>0$. By the assumption that $\mathbf{E} \in\left(D G L \mid E_{0}\right)$ relative to $T$, it follows that $x_{n} \rightarrow x$ in $S(\mathbf{E})$. Using (4.1) and the assumption that $\mathbf{E} \in\left(D^{\infty}\right)$, it now follows that $x_{n} \rightarrow x$ in $E_{0}+\infty E_{1}=E_{0}$ and consequently in the equivalent norm $\||\cdot|\| \mid$. This completes the proof of the proposition.

Corollary 4.6. If $\Lambda_{\phi}$ is a separable Lorentz space on $[0, \infty)$, and if $(\mathcal{M}, \tau)$ is a semi-finite von Neumann algebra then the space $\Lambda_{\phi}(\mathcal{M}, \tau)$ has an equivalent fully symmetric locally uniformly convex norm.

Proof. It follows from Theorem 3.6 that the Banach couple $\mathbf{E}=$ $\left(\Lambda_{\phi}(\mathcal{M}, \tau), \mathcal{M}\right) \in\left(D G L \mid\left(\Lambda_{\phi}(\mathcal{M}, \tau)\right)\right.$ relative to $(0, \infty)$, from Theorem 3.9, 
it follows that $\left(\Lambda_{\phi}(\mathcal{M}, \tau), \mathcal{M}\right) \in\left(D^{\infty}\right)$, and from Proposition 3.8, it follows that $\Lambda_{\phi}(\mathcal{M}, \tau)$ is Gagliardo complete with respect to the couple $\mathbf{E}$. The Corollary is now an immediate consequence of Proposition 4.5 preceding.

Suppose that $G$ is a symmetric Banach function space on $\mathbb{R}^{+}$. We recall that the fundamental function $\varphi_{G}$ of $G$ is defined by setting

$$
\varphi_{G}(t):=\left\|\chi_{[0, t)}\right\|_{G}, \quad t>0 .
$$

Let $\varphi$ be the least concave majorant of $\varphi_{G}$. It is shown in ([19], II.4, Theorem 5.5) that the Lorentz space $\Lambda_{\varphi}$ satisfies $\Lambda_{\varphi} \subseteq G$ and that

$$
\|x\|_{G} \leq\|x\|_{\Lambda_{\varphi}}, \quad x \in \Lambda_{\varphi} .
$$

Note that concavity of $\varphi$ implies that

$$
\varphi^{\prime}(t) \leq \varphi(1)
$$

a.e. for $t>1$.

Lemma 4.7. Let $G$ be a symmetric Banach function space on $\mathbb{R}^{+}$and suppose that $G \subseteq L_{1}[0, \infty)$. If

$$
\|x\|_{G}^{(1)}:=\left\|x^{*} \chi_{[0,1)}\right\|_{G}+\|x\|_{L_{1}[0, \infty)}, \quad x \in G,
$$

then the norms $\|\cdot\|_{G}$ and $\|\cdot\|_{G}^{(1)}$ are equivalent.

Proof. Since $G \subseteq L_{1}[0, \infty)$, there exists a constant $c>0$ such that

$$
\|x\|_{L_{1}[0, \infty)} \leq c\|x\|_{G}, \quad x \in G
$$

and consequently

$$
\|x\|_{G}^{(1)}=\left\|x^{*} \chi_{[0,1)}\right\|_{G}+\|x\|_{L_{1}[0, \infty)} \leq(1+c)\|x\|_{G}
$$

for all $x \in G$. To obtain the reverse estimate, let $\varphi$ be the least concave majorant of the fundamental function of $G$ and set

$$
x^{(1)}:=x^{*}(1) \chi_{[0,1)}+x^{*} \chi_{[1, \infty)}
$$

for all $x \in G$. Using (4.2), we observe that

$$
\begin{aligned}
\left\|x^{(1)}\right\|_{\Lambda_{\varphi}} & =\int_{[0, \infty)} x^{(1)}(t) d \varphi(t)=\int_{[0,1)} x^{*}(1) d \varphi(t)+\int_{[1, \infty)} x^{*}(t) d \varphi(t) \\
& \leq x^{*}(1) \varphi(1)+\int_{[1, \infty)} x^{*}(t) \varphi(1) d t=\varphi(1)\left\|x^{(1)}\right\|_{L_{1}[0, \infty)} .
\end{aligned}
$$


Consequently,

$$
\begin{aligned}
\|x\|_{G} & \leq\left\|x^{*} \chi_{[0,1)}+x^{(1)}\right\|_{G} \leq\left\|x^{*} \chi_{[0,1)}\right\|_{G}+\left\|x^{(1)}\right\|_{G} \leq\left\|x^{*} \chi_{[0,1)}\right\|_{G}+\left\|x^{(1)}\right\|_{\Lambda_{\varphi}} \\
& \leq\left\|x^{*} \chi_{[0,1)}\right\|_{G}+\varphi(1)\left\|x^{(1)}\right\|_{L_{1}[0, \infty)} \leq\left\|x^{*} \chi_{[0,1)}\right\|_{G}+\varphi(1)\|x\|_{L_{1}[0, \infty)}
\end{aligned}
$$

for all $x \in G$. It follows that

$$
\|x\|_{G} \leq \max (1, \varphi(1))\|x\|_{G}^{(1)}
$$

for all $x \in G$, and this completes the proof of the Lemma.

Theorem 4.8. If $G$ is a symmetric Banach function space on $\mathbb{R}^{+}$, then $G$ admits an equivalent symmetric locally uniformly convex norm if and only if $G$ is separable, that is, if and only if the norm on $G$ is order continuous.

Proof. The necessity follows from ([16], Corollary 1.4) and Proposition 2.6. To prove sufficiency, we let $\mathbf{L}$ denote the Banach couple $\left(L_{1}[0, \infty), L_{\infty}[0, \infty)\right)$ and note that $\mathbf{L}_{\Phi_{G}}=G$. We assume first that $G \nsubseteq L_{1}[0, \infty)$. It then follows from ([16], Theorem 4.2 (ii)) that the functional $\Phi_{G}$ is order-continuous on $Q$, and from ([16], Theorem 4.1(ii)(a)), it follows that the functional $\Phi_{G}$ is lower-semicontinuous on $Q$. By Theorem 3.6, $\mathbf{L} \in(D G L \mid G)$ relative to $T=(0, \infty)$. Theorem 4.2 now implies that there exists on $G$ an equivalent symmetric locally uniformly convex norm.

We may therefore assume that $G \subseteq L_{1}[0, \infty)$. If we set

$$
\|x\|_{1}:=\left[\|x\|_{L_{1}[0, \infty)}^{2}+\int_{0}^{\infty}\left(K(t, x) \min \left(1, t^{-2}\right)\right)^{2} d t\right]^{1 / 2}, \quad x \in L_{1}[0, \infty),
$$

with $K(t, x)=\int_{0}^{t} x^{*}(s) d s, t>0$, then $\|\cdot\|_{1}$ is an equivalent fully symmetric locally uniformly convex norm on $L_{1}[0, \infty)$, as shown in the proof of Proposition 4.5. Further, from Lemma 4.7, it is clear that the norm $\|\cdot\|_{G}^{(2)}$ given by setting

$$
\|x\|_{G}^{(2)}=\left(\left\|x^{*} \chi_{[0,1)]}\right\|_{G}^{2}+\|x\|_{1}^{2}\right)^{1 / 2}, \quad x \in G,
$$

is equivalent to $\|\cdot\|_{G}$. We now define the fully symmetric norm $\||\cdot|\|$ on $G$ by setting

$$
\begin{aligned}
\|\| x\|\| & =\left(\|x\|_{1}^{2}+\sum_{m=0}^{\infty} 2^{-m}\left\|x^{*} \chi_{\left[0,2^{-m}\right)}\right\|_{G}^{2}\right)^{1 / 2} \\
& =\left\|\left(\|x\|_{1},\left\|x^{*} \chi_{[0,1)}\right\|_{G}, \ldots, 2^{-m / 2}\left\|x^{*} \chi_{\left[0,2^{-m}\right)}\right\|_{G}, \ldots\right)\right\|_{l^{2}(\mathbb{N})}
\end{aligned}
$$


for all $x \in G$. It is easily seen that

$$
\|x\|_{G}^{(2)} \leq\|\| x \mid\|\leq \sqrt{2}\| x \|_{G}^{(2)}
$$

for all $x \in G$ and so the norm \|\|$\cdot \|$ is equivalent to the given norm $\|\cdot\|_{G}$ on $G$. We now show that the norm $\|\mid \cdot\|$ is locally uniformly convex. To this end suppose that $x, x_{n} \in G, n=1,2, \ldots$ satisfy $\left\|\left|x_{n}\|\| \rightarrow\left\||| x\left|\left\|,||\left|x+x_{n}\right|\right\|\right| \rightarrow 2|\|x\||\right.\right.\right.$. Using the uniform convexity and as well the uniform strong majorant property of the Hilbert space $l_{2}(\mathbb{N})$, standard arguments show that

$$
\left\|x_{n}\right\|_{1} \rightarrow\|x\|_{1}, \quad\left\|x_{n}+x\right\|_{1} \rightarrow 2\|x\|_{1}
$$

and that

$$
\left\|x_{n}^{*} \chi_{\left[0,2^{-m}\right)}\right\|_{G} \rightarrow_{n}\left\|x \chi_{\left[0,2^{-m}\right)}\right\|_{G}
$$

for all $m=1,2, \ldots$. Set $y_{n}=x_{n}-x, n=1,2, \ldots$. Since the norm $\|\cdot\|_{1}$ is locally uniformly convex, it follows that $\left\|y_{n}\right\|_{1} \rightarrow 0$. Since

$$
y_{n}^{*}(a) \chi_{[0, a)} \leq y_{n}^{*}, \quad n=1,2, \ldots
$$

for all $a>0$, it follows also that

$$
y_{n}^{*}(a) \rightarrow_{n} 0
$$

for each fixed $a>0$. To show that $\left\|y_{n}\right\|_{G}^{(2)} \rightarrow 0$, it follows from the definition of $\|\cdot\|_{G}^{(2)}$ that it suffices to show that $\left\|y_{n}^{*} \chi_{[0,1)}\right\|_{G} \rightarrow 0$. Suppose then that $\epsilon>0$ is given. By order continuity of the norm $\|\cdot\|_{G}$, there exists a natural number $m$ such that $\left\|x^{*} \chi_{\left[0,2^{-m}\right)}\right\|_{G}<\epsilon$, and so, via (4.4)

$$
\left\|x_{n}^{*} \chi_{\left[0,2^{-m}\right)}\right\|_{G}<\epsilon
$$

for all sufficiently large $n$. Now observe that

$$
\begin{aligned}
\left\|y_{n}^{*} \chi_{[0,1)}\right\|_{G} & \leq\left\|y_{n}^{*} \chi_{\left[0,2^{-m}\right)}\right\|_{G}+\left\|y_{n}^{*} \chi_{\left[2^{-m}, 1\right)}\right\|_{G} \\
& \leq\left\|x_{n}^{*} \chi_{\left[0,2^{-m}\right)}\right\|_{G}+\left\|x^{*} \chi_{\left[0,2^{-m}\right)}\right\|_{G}+y_{n}^{*}\left(2^{-m}\right)\left\|\chi_{\left[2^{-m}, 1\right)}\right\|_{G} .
\end{aligned}
$$

From (4.5) and (4.6), it now follows that

$$
\left\|y_{n}^{*} \chi_{[0,1)}\right\|_{G}<3 \epsilon
$$

for all sufficiently large $n$, and this suffices to complete the proof of the Theorem. 
Corollary 4.9. Let $G$ be a fully symmetric Banach function space on $[0, \alpha), \quad 0<\alpha \leq \infty$ and let $\mathbf{E}$ be a Banach couple. If $G$ is separable and if $\mathbf{E} \in\left(D G L \mid \mathbf{E}_{G}\right)$ relative to $T=(0, \alpha)$ then the interpolation space $\mathbf{E}_{G}$ has an equivalent fully symmetric locally uniformly convex norm if

(i) $\alpha<\infty$, or if $\alpha=\infty$ and $G \nsubseteq L_{1}$;

(ii) $\alpha=\infty$ and $E_{1} \subseteq E_{0}$;

(iii) $\alpha=\infty$ and $\mathbf{E} \in\left(D^{\infty}\right)$.

Proof. It follows from Corollary 4.3 if $\alpha<\infty$ and from Theorem 4.8 preceding if $\alpha=\infty$ that $G$ has an equivalent (fully) symmetric locally uniformly convex norm $\||\cdot \||$. The corollary now follows immediately from Theorem 2.3 applied to $(G,|||\cdot| \mid)$.

If we combine Theorem 3.6 and Theorem 3.9 with the preceding Corollary 4.9 applied to the couple $\mathbf{L}=\mathbf{L}(\mathcal{M}, \tau)$, where $(\mathcal{M}, \tau)$ is an arbitrary semifinite von Neumann algebra, then we obtain the following extension of Corollary 4.3.

Corollary 4.10. If $(\mathcal{M}, \tau)$ is an arbitrary semifinite von Neumann algebra and if $E$ is a separable symmetric Banach function space on $[0, \tau(\mathbf{1}))$, then the space $E(\mathcal{M}, \tau)$ has an equivalent fully symmetric locally uniformly convex norm.

The result of the preceding Corollary is new, even in the commutative setting of separable, symmetric Banach function spaces on the positive semiaxis. In this case, the main result of [17] does indeed yield the existence of an equivalent lattice norm. This lattice norm, however, is rearrangementinvariant only in the case of symmetric spaces on the unit interval $[0,1]$. The preceding Corollary 4.10 also implies a renorming theorem due to Sedaev $[$ Se1,2] (see ([7], Theorem 1.5)) as well as its non-commutative extension given in ([6], Corollary 2.10). In the non-commutative setting, Corollary 4.10 extends Corollary 2.2 of [8] from non-atomic finite von Neumann algebras to general semifinite von Neumann algebras. Finally, we note that Corollary 4.10 can be derived from Theorem 4.8 and ([8], Theorem 3.1). However, it seems that the approach of the present paper gives greater clarity and structural insight.

\section{References}

[1] J. Arazy, More on convergence in unitary matrix spaces, Proc. Amer. Math. Soc., 83 (1981), 44-48.

[2] J. Arazy, On the geometry of the unit ball of unitary matrix spaces, Integral Eq. Oper. Th., 4 (1981), 151-171. 
[3] B. Beauzamy, Proprietes geometriques des Espaces d'Interpolation, Seminaire Maurey Schwartz 1975-1975, Expose 14, Ecole Polytechnique, Paris.

[4] C. Bennett and R. Sharpley, Interpolation of Operators, Academic Press, 1988.

[5] J. Bergh and J. Lofstrom, Interpolation Spaces, Vol. 223, Springer, Berlin-Heidelberg-New York, 1976.

[6] V.I. Chilin, P.G. Dodds, and F.A. Sukochev, The Kadec-Klee property in symmetric spaces of measurable operators, Israel J. Math., 97 (1997), 203-219.

[7] V.I. Chilin, P.G. Dodds, A.A. Sedaev and F.A.Sukochev, Characterization of Kadec-Klee properties in symmetric spaces of measurable functions, Trans. Amer. Math. Soc., 348 (1996), 4895-4918.

[8] V.I. Chilin, A.V. Krygin and F.A. Sukochev, Local uniform and uniform convexity of non-commutative symmetric spaces of measurable operators, Math. Proc. Camb. Philos. Soc., 111 (1992), 355-368.

[9] V.I. Chilin and F.A. Sukochev, Weak convergence in non-commutative symmetric spaces, J. Operator Theory, 31 (1994), 35-65.

[10] M.M. Day, Normed Linear Spaces, Ergebnisse der Math., Vol. 21, Springer, 1973.

[11] J. Diestel, Geometry of Banach Spaces-Selected Topics, Vol. 485, Springer Lecture Notes in Mathematics, 1975.

[12] P.G. Dodds, T.K. Dodds and B. de Pagter, Non-commutative Banach function spaces, Math. Z., 201 (1989), 583-597.

[13] P.G. Dodds, T.K. Dodds and B. de Pagter, Fully symmetric operator spaces, Integr. Equat. Oper. Th., 15 (1992), 942-972.

[14] P.G. Dodds, T.K. Dodds and B. de Pagter, Non-commutative Köthe duality, Trans. Amer. Math. Soc., 339 (1993), 717-750.

[15] P.G. Dodds, T.K. Dodds and F.A. Sukochev, Lifting of Kadec-Klee properties to symmetric spaces of measurable operators, Proc. Amer. Math. Soc., 125 (1997), 1457-1467.

[16] P.G. Dodds, T.K. Dodds, A.A. Sedaev and F.A. Sukochev, Local uniform convexity and Kadec-Klee properties in K-interplation spaces I: General Theory, J. Function Spaces Appl. 2 (2004) 125-173.

[17] W.J. Davis, N. Ghoussoub and J. Lindenstrauss, A lattice renorming theorem and applications to vector-valued processes, Trans. Amer. Math. Soc., 263 (1981), 531-540.

[18] T. Fack and H. Kosaki, Generalized s-numbers of $\tau$-measurable operators, Pacific J. Math., 123 (1986), 269-300. 
[19] S.G. Krein, Ju.I. Petunin and E.M. Semenov, Interpolation of linear operators, Translations of Mathematical Monographs, Amer. Math. Soc., 54 (1982).

[20] J. Lindenstrauss and L. Tzafriri, Classical Banach Spaces I, SpringerVerlag, 1977.

[21] J. Lindenstrauss and L. Tzafriri, Classical Banach Spaces II, SpringerVerlag, 1979.

[22] E. Nelson, Notes on non-commutative integration, J. Functional Anal., 15 (1974), 103-116.

[23] A.A. Sedaev, On $(H)$-property in symmetric spaces, Teor. Func. Func. Anal. Prilozh., 11 (1970), 67-80. (Russian)

[24] A.A. Sedaev, On weak and strong convergence in interpolation spaces, Trudy 6 zimney shkoly po mat. programm.i smez. vopr, CEMI, Moscow (1975), 245-267. (Russian)

[25] A.A. Sedaev and F.A. Sukochev, The Kadets-Klee property and local uniform convexity properties in Interpolation Spaces of the K-Method, Russian Acad. Sci. Dokl. Math., 50 (1995), 343-347.

[26] S. Stratila, Modular Theory in Operator Algebras, Abacus Press, Tunbridge Wells, 1981.

[27] S. Stratila and L. Zsidó, Lectures on Von Neumann Algebras, Editura and Abacus Press, 1979.

[28] M. Takesaki, Theory of Operator Algebras I, Springer-Verlag, New YorkHeidelberg-Berlin, 1979.

[29] M. Terp, L $L_{p}$-spaces associated with von Neumann algebras, Notes, Copenhagen Univ., 1981.

P. G. Dodds, T. K. Dodds, F. A. Sukochev

School of Informatics and Engineering

Flinders University, Bedford Park, SA 5042

Australia

(E-mails : peter,theresa,sukochev@infoeng.finders.edu.au)

A. A. Sedaev

Department of Mathematics

Voronezh State University

of Architecture and Civil Engineering

394006 Voronezh, 20-letiya Oktyabrya 84

Russia

(E-mail : sed@vmail.ru)

(Received : August 2003) 


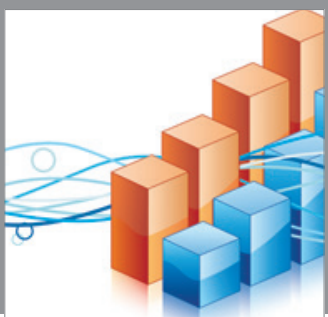

Advances in

Operations Research

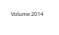

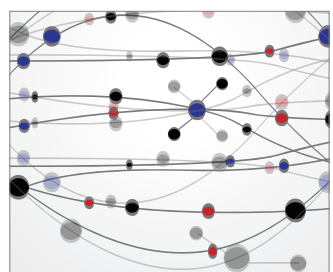

\section{The Scientific} World Journal
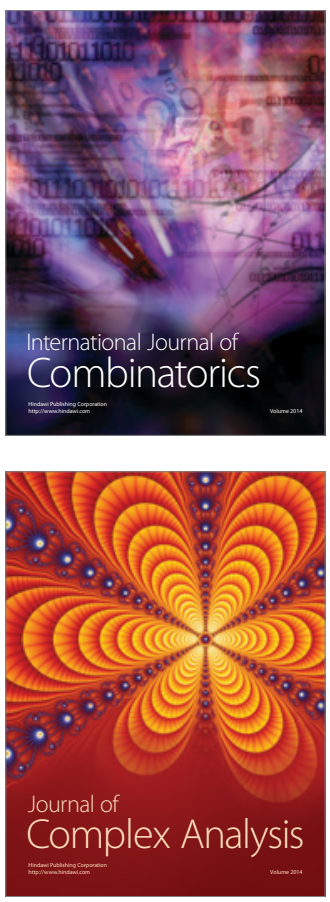

International Journal of

Mathematics and

Mathematical

Sciences
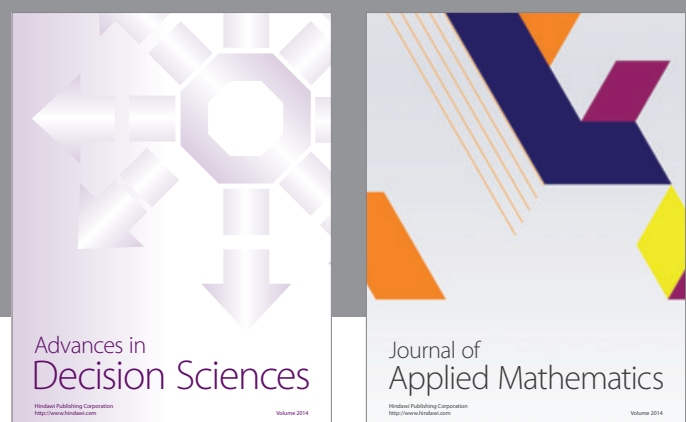

Journal of

Applied Mathematics
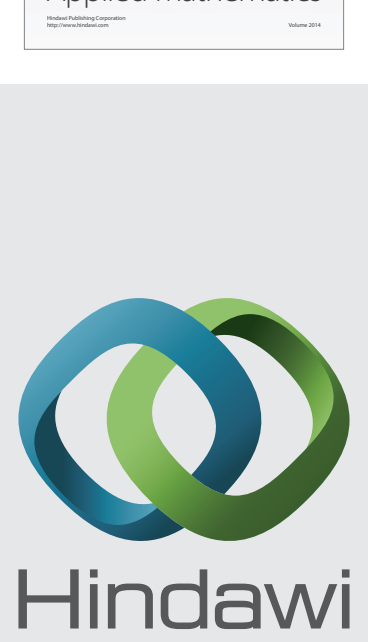

Submit your manuscripts at http://www.hindawi.com
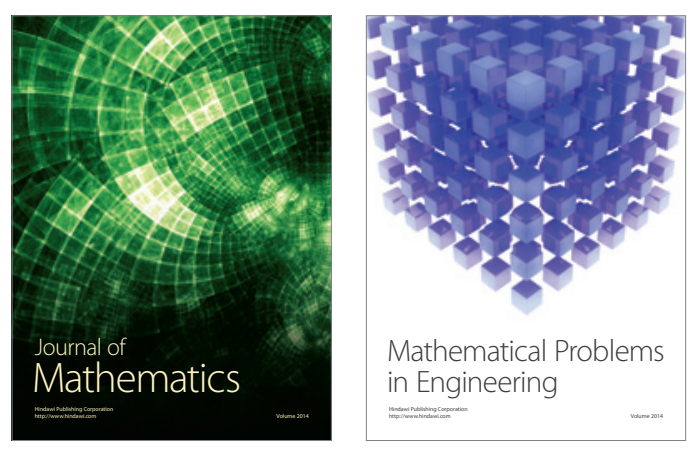

Mathematical Problems in Engineering
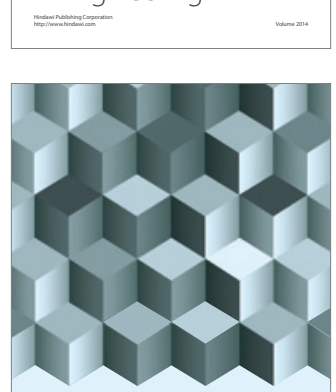

Journal of

Function Spaces
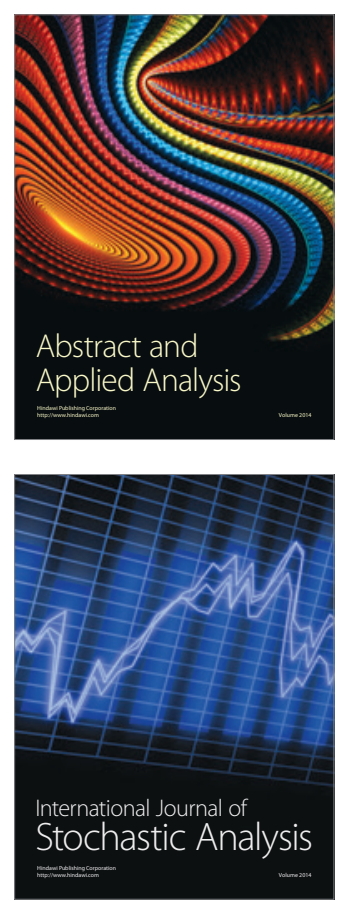

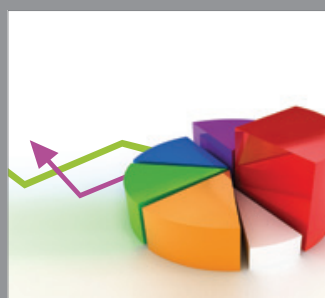

ournal of

Probability and Statistics

Promensencen
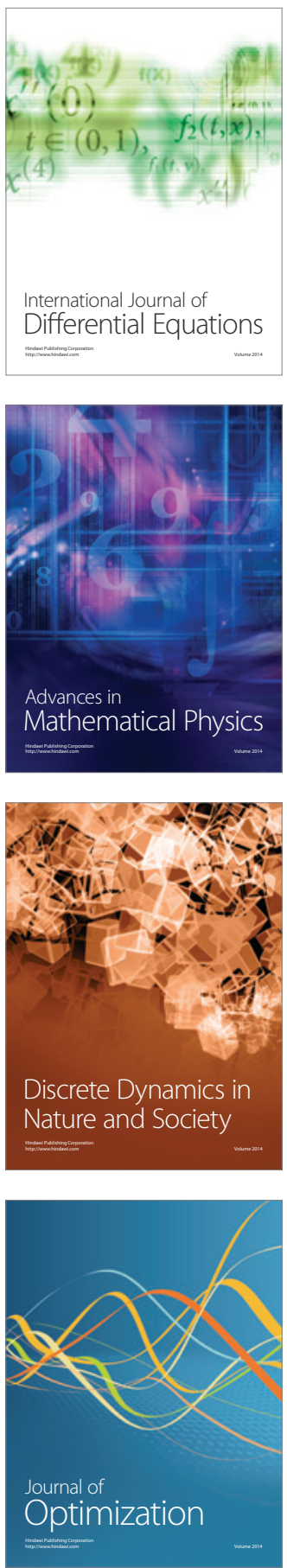